\title{
LHC predictions from a tevatron anomaly in the top quark forward-backward asymmetry
}

\author{
Yang Bai, JoAnne L. Hewett, Jared Kaplan and Thomas G. Rizzo \\ SLAC National Accelerator Laboratory, \\ 2575 Sand Hill Road, Menlo Park, CA 94025, U.S.A. \\ E-mail: yangbai@slac.stanford.edu, hewett@slac.stanford.edu, \\ jaredk@slac.stanford.edu, rizzo@slac.stanford.edu
}

ABstRACT: We examine the implications of the recent CDF measurement of the top-quark forward-backward asymmetry, focusing on a scenario with a new color octet vector boson at 1-3 TeV. We study several models, as well as a general effective field theory, and determine the parameter space which provides the best simultaneous fit to the CDF asymmetry, the Tevatron top pair production cross section, and the exclusion regions from LHC dijet resonance and contact interaction searches. Flavor constraints on these models are more subtle and less severe than the literature indicates. We find a large region of allowed parameter space at high axigluon mass and a smaller region at low mass; we match the latter to an $\mathrm{SU}(3)_{1} \times \mathrm{SU}(3)_{2} / \mathrm{SU}(3)_{c}$ coset model with a heavy vector-like fermion. Our scenario produces discoverable effects at the LHC with only $1-2 \mathrm{fb}^{-1}$ of luminosity at $\sqrt{s}=7-8 \mathrm{TeV}$. Lastly, we point out that a Tevatron measurement of the $b$-quark forwardbackward asymmetry would be very helpful in characterizing the physics underlying the top-quark asymmetry.

Keywords: Beyond Standard Model, Heavy Quark Physics

ARXIV EPRINT: 1101.5203 


\section{Contents}

1 Introduction 1

2 An axigluon? $\quad 3$

2.1 The $t \bar{t}$ cross section 4

2.2 Dijet resonance searches 5

2.3 Dijet contact interaction searches 5

$\begin{array}{lll}2.4 & \text { Phenomenological fit } & 7\end{array}$

3 Axigluon models $\quad 8$

3.1 The minimal axigluon model $\quad 9$

$\begin{array}{lll}3.2 & \text { A simplified three-site model } & 12\end{array}$

$\begin{array}{ll}3.3 \text { A two-site model with a new vector-like quark } & 14\end{array}$

$\begin{array}{lll}4 & \text { Flavor constraints } & 17\end{array}$

5 Signatures at the LHC and Tevatron $\quad 22$

$\begin{array}{lll}5.1 & \text { Dijet resonances } & 22\end{array}$

$\begin{array}{lll}5.2 & \text { Top-anti-top resonances } & 22\end{array}$

5.3 Production in association with a weak gauge boson 24

5.4 Bottom quark forward-backward asymmetry 24

$\begin{array}{llr}6 & \text { Discussion and conclusions } & 26\end{array}$

\section{Introduction}

CDF recently announced a $3.4 \sigma$ deviation from the Standard Model (SM) in a measurement of the $t \bar{t}$ forward-backward asymmetry [1]. Their study updates previous analyses that observed deviations of roughly $2 \sigma[2,3]$, but they also go further, demonstrating that the $t \bar{t}$ asymmetry is dominated by events with large invariant mass $M_{t \bar{t}}$. This rise in the asymmetry at high energy could be interpreted as further evidence of a new physics signal characterized by energies at or above the weak scale. In this work we will investigate explanations of the asymmetry based on new physics [4-9, 11-15], examine what can be done next at the Tevatron and the LHC to either rule out or confirm as well as characterize models that explain the anomaly.

An important first question is whether we should consider models that are wholly responsible for the asymmetry, or models where interference between new physics and QCD generates the asymmetry. After unfolding their acceptance, CDF observed [1] a parton-level asymmetry $A_{F B}^{t \bar{t}}=0.475 \pm 0.114$ in $t \bar{t}$ events with $M_{t \bar{t}}>450 \mathrm{GeV}$ and in the $t \bar{t}$ center of mass frame (see table 1), compared to the SM prediction of $A_{F B}^{t \bar{t}}(\mathrm{SM})=$ 
$0.088 \pm 0.013$. This is a large effect. If new physics alone generates the forward-backward asymmetry, a straightforward interpretation of these numbers suggests that new physics must be responsible for roughly half of all $t \bar{t}$ production above $450 \mathrm{GeV}$, even in a scenario where the new physics yields a maximal forward-backward asymmetry! However, a new heavy particle produced in the $s$-channel will have to contend with searches for resonances and contact interactions at both the Tevatron and the LHC [12]. For this reason, we will focus on models where the new physics interferes with QCD to produce the asymmetry, allowing for a smaller total contribution to production rates from new physics.

In order to interfere with SM top production, new physics processes must produce $t \bar{t}$ pairs in a state with the same quantum numbers as in QCD production. This means that the $t \bar{t}$ must form a color octet, Lorentz vector state, so if there is a new particle in the $s$ channel coupling to tops, it must be a heavy color octet vector boson. ${ }^{1}$ Such a 'heavy gluon partner' $G^{\prime}$ can arise in a wide variety of models [16], including top-color models $[17,18]$, technicolor [19], warped extra dimensions [20, 21], universal extra dimensions [22] and chiral color [23-25]. As we will discuss below, $G^{\prime}$ particles are constrained by flavor physics and collider searches, although we disagree with some exclusion claims in the literature. For convenience we will present a phenomenological low-energy effective field theory and some simple coset models, and we will ignore the issue of anomaly cancellation. Throughout we will use the term 'axigluon' as a shorthand for 'color octet vector boson'.

New physics produced in the $t$-channel [7] could also generate a substantial forwardbackward asymmetry. Naively, one might imagine that a $t$-channel contribution would not rise with energy, but in fact $t$-channel physics produces a large energy dependence that might fit the CDF results nicely. However, producing $t \bar{t}$ pairs via a new $t$-channel process generates non-trivial flavor issues; moreover, new $t$-channel effects already have significant tension with Tevatron data as they give rise to a large number of like-sign top pairs [7]. Since the LHC is a $p p$ collider, a new $t$-channel state could be discovered soon by searches for like-sign tops. We will not discuss these models any further here.

Future studies at both the Tevatron and the LHC could shed light on the nature of the $t \bar{t}$ asymmetry. The Tevatron could provide a great deal of insight into the physics responsible for the $t \bar{t}$ asymmetry by studying a potential $b \bar{b}$ asymmetry, using semi-leptonic $b$ decays to tag the sign of $b$-jets. If the heavy color octet $G^{\prime}$ has large enough couplings to generate a sizable asymmetry, it should be visible in dijet resonance or contact interaction searches this year (with $1-2 \mathrm{fb}^{-1}$ of luminosity) at the LHC. In fact, we will see below that a sizable portion of the possible parameter space has already been excluded by the LHC! Measurements of the $t \bar{t}$ production cross section are also sensitive to new physics, and a new $G^{\prime}$ could be discovered as a resonance decaying to $t \bar{t}$. Fixing the new physics contribution to $A_{F B}^{t \bar{t}}$, we will see that dijet and $t \bar{t}$ searches are complementary, as the product of the $G^{\prime}$ coupling to light quarks and tops must essentially be held fixed to account for the asymmetry. Whatever the verdict, in all likelihood new physics explanations for the asymmetry will be much better understood after the next year of operations at the LHC.

\footnotetext{
${ }^{1}$ In principle, colored particles with spin $>1$ might also interfere off-shell, but roughly speaking, this is due to the contribution of the vector particles they 'eat' to become massive.
} 


\begin{tabular}{|c|c|c|}
\hline Selection & $M_{t \bar{t}}<450 \mathrm{GeV}$ & $M_{t \bar{t}}>450 \mathrm{GeV}$ \\
\hline Parton Level Data & $-0.116 \pm 0.146 \pm 0.047$ & $0.475 \pm 0.101 \pm 0.049$ \\
\hline Selection & $|\Delta y|<1.0$ & $|\Delta y|>1.0$ \\
\hline Parton Level Data & $0.026 \pm 0.104 \pm 0.056$ & $0.611 \pm 0.210 \pm 0.147$ \\
\hline
\end{tabular}

Table 1. Unfolded parton level data for $A_{F B}^{t \bar{t}}$ from the CDF study [1].

The rest of this paper is organized as follows. Section 2 introduces an effective field theory for a heavy color octet vector boson and then discusses various collider bounds that constrain it. In section 3 we introduce specific coset models for $G^{\prime}$, and define the parameter space for each model that provides a fit to the CDF asymmetry data at $90 \%$ and 95\% C.L. We also show how the constraints from other collider data $\left(\sigma_{t \bar{t}}\right.$, dijet resonance, and dijet contact interaction searches) impact this parameter space. In section 4 we explore the contributions in the flavor sector from our models and show that meson mixing does not necessarily restrict the relevant parameter space. Section 5 delineates various signatures of the heavy color octet at the LHC. Lastly, section 6 contains our conclusions.

\section{$2 \quad$ An axigluon?}

Our goal is to examine if a new color octet vector boson $G^{\prime}$ can explain the discrepancy in the recently measured $t \bar{t}$ forward-backward asymmetry. To this end, let us begin with a general low-energy effective Lagrangian coupling $G^{\prime}$ to the SM quarks. To suppress dangerous FCNC effects it will prove advantageous to assume universal vector and axial couplings to the first two generations, $g_{V}^{q}$ and $g_{A}^{q}$, while the couplings to the top quark and bottom quark will be $g_{V}^{t}$ and $g_{A}^{t}$. For convenience we have rescaled these couplings in terms of the strong coupling $g_{s}$. The tree-level differential cross section for $q \bar{q} \rightarrow t \bar{t}$ will be $[8,26]$

$$
\begin{aligned}
& \frac{d \hat{\sigma}^{q \bar{q} \rightarrow t \bar{t}}}{d \cos \theta^{*}}=\alpha_{s}^{2} \frac{\pi \sqrt{1-4 m^{2}}}{9 \hat{s}} \times \\
& \times\left[\left(1+4 m^{2}+c^{2}\right)\left(1-\frac{2 g_{V}^{q} g_{V}^{t} \hat{s}\left(M_{G^{\prime}}^{2}-\hat{s}\right)}{\left(\hat{s}-M_{G^{\prime}}^{2}\right)^{2}+M_{G^{\prime}}^{2} \Gamma_{G}^{2}}+\frac{g_{V}^{t 2}\left(g_{V}^{q 2}+g_{A}^{q 2}\right) \hat{s}^{2}}{\left(\hat{s}-M_{G^{\prime}}^{2}\right)^{2}+M_{G^{\prime}}^{2} \Gamma_{G}^{2}}\right)\right. \\
& +\left(1-4 m^{2}+c^{2}\right) g_{A}^{t 2}\left(g_{V}^{q 2}+g_{A}^{q 2}\right) \frac{\hat{s}^{2}}{\left(\hat{s}-M_{G^{\prime}}^{2}\right)^{2}+M_{G^{\prime}}^{2} \Gamma_{G}^{2}} \\
& \left.-4 g_{A}^{q} g_{A}^{t} c\left(\frac{\hat{s}\left(M_{G^{\prime}}^{2}-\hat{s}\right)}{\left(\hat{s}-M_{G^{\prime}}^{2}\right)^{2}+M_{G^{\prime}}^{2} \Gamma_{G}^{2}}-2 g_{V}^{q} g_{V}^{t} \frac{\hat{s}^{2}}{\left(\hat{s}-M_{G^{\prime}}^{2}\right)^{2}+M_{G^{\prime}}^{2} \Gamma_{G}^{2}}\right)\right]
\end{aligned}
$$

where $m^{2}=m_{t}^{2} / \hat{s}, c=\sqrt{1-4 m^{2}} \cos \theta^{*}$, and $\theta^{*}$ is the angle between the top quark and the incoming quark in the center of mass frame. The forward-backward asymmetry arises solely from the last line in this equation, so to obtain a positive asymmetry we must have $g_{A}^{q} g_{A}^{t}<0$. 
We will be considering $M_{G^{\prime}} \gtrsim 1 \mathrm{TeV}$, so at the Tevatron we have $\hat{s}<M_{G^{\prime}}^{2}$ over most of the support of the parton distribution functions. In this limit the interference term between the QCD and resonance contributions dominates over the pure resonance term, and we expect the following qualitative features:

- the asymmetry is directly proportional to $\left(-g_{A}^{q} g_{A}^{t}\right)$

- relative to the pure QCD cross section, the asymmetric term grows $\propto \hat{s}$, so the asymmetry will grow with the invariant mass squared $M_{t \bar{t}}^{2}$

- the total $t \bar{t}$ cross section at low energies will decrease with increasing $g_{V}^{q} g_{V}^{t}$.

Additionally, we see that the sign and magnitude of $g_{V}^{q} g_{V}^{t}$ determine the behavior of the asymmetry at high energies, and that for larger $g_{V}^{q} g_{V}^{t}$, our qualitative discussion based on the parametric expansion in $\hat{s} / M_{G^{\prime}}^{2}$ breaks down more quickly.

Axigluons face constraints from flavor-changing neutral currents (FCNCs), from $t \bar{t}$ resonance searches, and from measurements of the total $t \bar{t}$ cross section. Furthermore, any axigluon that couples to $q \bar{q}$ is subject to constraints from dijet resonance and dijet contact interaction searches - not just at the Tevatron but at the LHC! As we will see, the strongest constraints on our models will come from the LHC, and the prospects of a discovery or a definitive exclusion this year are extremely good. This is striking proof that we have entered the LHC era!

Before considering the details, note that at least qualitatively, once we fix $g_{A}^{q} g_{A}^{t}$, the $t \bar{t}$ and dijet constraints will be orthogonal. Increasing $\left|g_{A}^{q} g_{A}^{t}\right|$ increases the asymmetry, but eventually one runs afoul of constraints; one might avoid altering the $t \bar{t}$ cross section by increasing $g_{V}^{q} g_{V}^{t}$, but only at the expense of decreasing the asymmetry itself. Now let us discuss the restrictions from the $t \bar{t}$ cross section and the dijet searches; we will leave a discussion of flavor to section 4 .

\subsection{The $t \bar{t}$ cross section}

Theoretical predictions [27] of the $t \bar{t}$ cross section have been verified at the Tevatron [28]. An axigluon will modify both the total $t \bar{t}$ cross section and its shape as a function of the invariant mass $M_{t \bar{t}}$, so Tevatron $t \bar{t}$ measurements restrict axigluon models. However, we cannot set a precise constraint without a more detailed understanding of the experimental and theoretical details. The current $t \bar{t}$ resonance searches at the Tevatron [29-33] have not set a limit on a possible resonance with mass beyond $\sim 800 \mathrm{GeV}$ due to a lack of statistics in their published analyses.

In our analysis below, we will display contours corresponding to an axigluon contribution of $20 \%$ and $40 \%$ to the total QCD $t \bar{t}$ cross section for $M_{t \bar{t}}>450 \mathrm{GeV}$. These contours should be viewed as a suggestion of the Tevatron sensitivity, and not as hard bounds. One might instead consider using a larger cut on the top invariant mass. This would result in a larger relative axigluon contribution to the cross section, but would compete with the uncertainties on the QCD cross section which grow rapidly at higher invariant mass. After some experimentation, we chose this $M_{t \bar{t}}>450 \mathrm{GeV}$ requirement as a reasonable and conservative compromise between these two factors. 
For illustrative purposes, we show the $M_{t \bar{t}}$ distributions in the upper panel of figure 1. With the exception of the magenta line, the other curves on the histogram represent a $1 \mathrm{TeV}$ axigluon. For the red(blue) lines, the coupling of the axigluon is of QCD strength and $\Gamma / M=0.1(0.2)$ has been assumed. For the green(cyan) histogram, the coupling strengths of the axigluon have been doubled and now $\Gamma / M=0.4(0.8)$ has been assumed. One can see that a $1 \mathrm{TeV}$ axigluon with a narrow width will appear as a $t \bar{t}$ resonance.

\subsection{Dijet resonance searches}

CMS recently performed a search for narrow dijet resonances [34], employing an integrated luminosity of $2.9 \mathrm{pb}^{-1}$. This search has ruled out colorons and axigluons (with couplings $g_{V}^{q, t}=1$ or $g_{A}^{q, t}=1$ respectively, in our notation) in the mass intervals $0.50<M_{G^{\prime}}<$ $1.17 \mathrm{TeV}$ and $1.47<M_{G^{\prime}}<1.52 \mathrm{TeV}$. In the narrow width approximation the production cross section for $q \bar{q} \rightarrow G^{\prime} \rightarrow q \bar{q}$ is

$$
\frac{\sigma \times B R\left(q \bar{q} \rightarrow G^{\prime} \rightarrow j j\right)}{\sigma \times B R(\text { coloron })}=\frac{6}{5}\left(\left|g_{V}^{q}\right|^{2}+\left|g_{A}^{q}\right|^{2}\right) \frac{4\left(\left|g_{V}^{q}\right|^{2}+\left|g_{A}^{q}\right|^{2}\right)+\left(\left|g_{V}^{t}\right|^{2}+\left|g_{A}^{t}\right|^{2}\right)}{4\left(\left|g_{V}^{q}\right|^{2}+\left|g_{A}^{q}\right|^{2}\right)+2\left(\left|g_{V}^{t}\right|^{2}+\left|g_{A}^{t}\right|^{2}\right)},
$$

where we have related it to the cross section for a coloron with coupling $g_{s}$, and we have assumed an identical coupling to bottom and top quarks. Using the CMS 95\% C.L. exclusion upper limit on dijet resonance production, $\sigma \times A$ (where $A$ is the acceptance), for the $q \bar{q}$ initial state and then rescaling the cross sections of colorons and axigluons in figure 4 of ref. [34], we can estimate the corresponding dijet resonance constraints [35].

The dijet resonance searches are based on the narrow width approximation, so they do not constrain axigluons with large widths. To give an idea of when the resonance has become too broad for this search, in the lower panel of figure 1 we show a sample of LHC dijet invariant mass distributions for the signal and QCD background. As one can see from this figure, for $\Gamma / M \simeq 0.1$ (the red line) the signal shows a bump-like feature on top of the QCD background (the black line). However, for $\Gamma / M \gtrsim 0.2$ (the blue line) the bump is much less visible. We will thus assume in what follows that the narrow resonance search becomes inapplicable when $\Gamma / M \gtrsim 0.2$.

\subsection{Dijet contact interaction searches}

Searches for quark contact interactions can be sensitive to broad axigluons via dijet angular distributions. This follows because LO QCD dijet production is dominated by $t$-channel Rutherford-like scattering. For a small production angle $\theta^{*}$ in the center of mass frame, the QCD differential cross section behaves as $d \hat{\sigma} / d \cos \theta^{*} \sim 1 / \sin ^{4}\left(\theta^{*} / 2\right)$. Defining the variable $\chi=\left(1+\cos \theta^{*}\right) /\left(1-\cos \theta^{*}\right)$ and considering the limit $\chi \rightarrow \infty$, one finds that $d \hat{\sigma} / d \chi \sim$ constant. On the other hand, the differential distribution for dijets arising from resonance production is not flat in $\chi$ but instead has a peak at small values of $\chi$. By comparing the corresponding shapes of the signal and background distributions in $\chi$, ATLAS has excluded LL quark contact interactions ${ }^{2}$ with a compositeness scale below $3.4 \mathrm{TeV}$ at $95 \%$

\footnotetext{
${ }^{2}$ The use of a left-left contact interaction is a common convention or benchmark; the limits on a L-R or $\mathrm{R}-\mathrm{R}$ interaction would be similar in magnitude.
} 

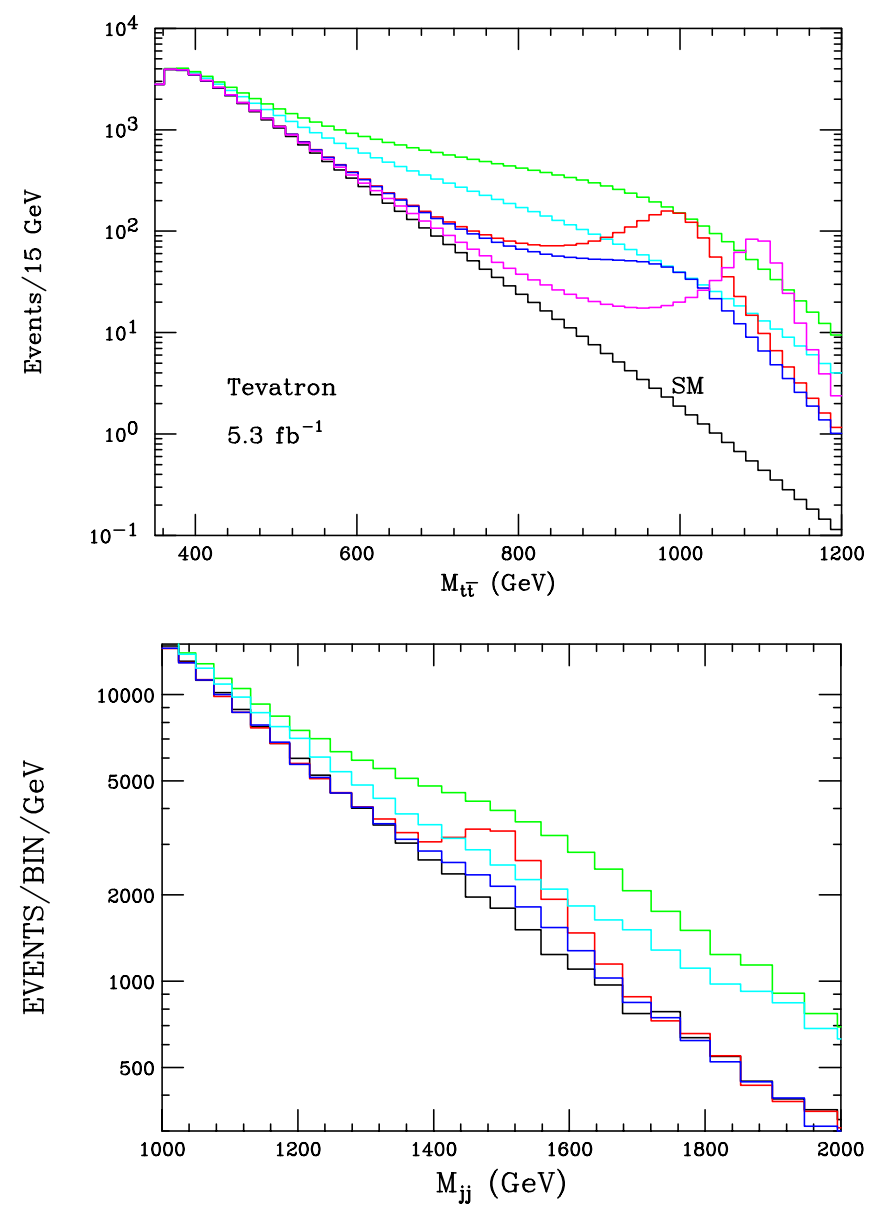

Figure 1. Upper panel: the axigluon contribution to the $t \bar{t}$ invariant mass distribution at the Tevatron. No $t$-quark branching fractions or reconstruction efficiencies have been employed. The magenta curve represents a model we will discuss later in section 3.3 with parameter values $\theta=30^{\circ}$ and $M_{G^{\prime}}=1100 \mathrm{GeV}$. The black histogram is the QCD prediction whereas the red(blue) histogram assumes the existence of a $1.0 \mathrm{TeV}$ axigluon with QCD strength couplings with $\Gamma / M \simeq 0.1(0.2)$. For the green(cyan) histogram, the coupling strengths of the axigluon have been doubled and now $\Gamma / M \simeq 0.4(0.8)$ has been assumed. The CTEQ6.6M PDFs were employed. Lower panel: axigluon contribution to the dijet pair production cross section at the LHC assuming $\sqrt{s}=8 \mathrm{TeV}$ and an integrated luminosity of $\mathrm{L}=5 \mathrm{fb}^{-1}$ after applying the cuts $\left|\eta_{j}\right|<0.5, p_{T j}>200 \mathrm{GeV}$ and CMS detector smearing. All other four colored histograms are for a $1.5 \mathrm{TeV}$ axigluon with the same $\Gamma / M$ ratios as the upper panel.

C.L. [36]. To set corresponding limits on our model parameter space, we require that the new physics contribution plus the SM background not exceed the ATLAS data at 95\% C.L. for their chosen mass intervals $800<m_{j j}<1200 \mathrm{GeV}$ and $m_{j j}>1200 \mathrm{GeV}$. Only the first four ATLAS bins with $1<\chi<3.32$ are included in this analysis, which we base on the optimized sensitivity to quark contact interactions given in ref. [36]. As an example of this search in action, the constraints from dijet contact interaction searches are shown as the solid dark yellow line of figure 2, which refers to the effective field theory of section 2 . 

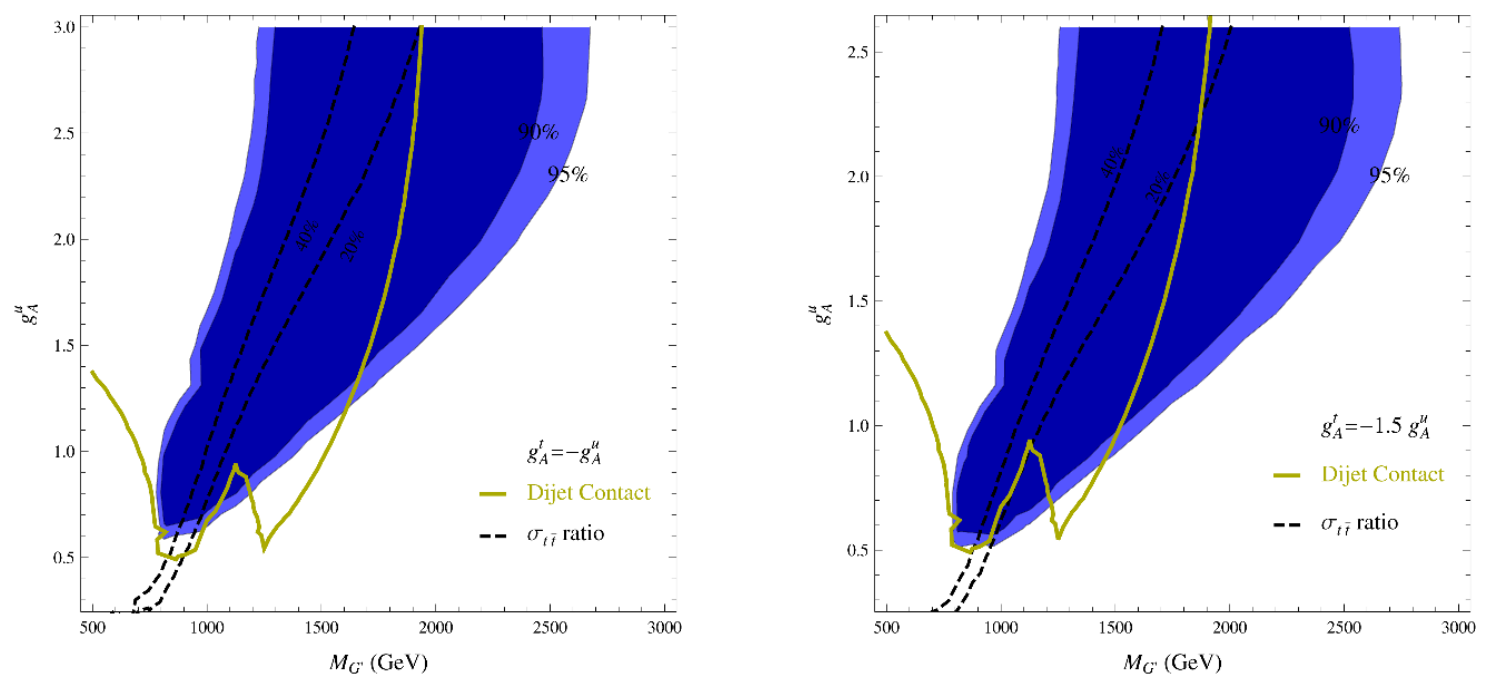

Figure 2. Left panel: the fit for the phenomenological model of eq. 2.1 with $g_{A}^{q}>0, g_{A}^{t}<0$, $g_{V}^{q, t}=0$ and a fixed ratio $g_{A}^{t} / g_{A}^{q}=-1$. The region above and to the left of the dark yellow solid line is excluded by dijet contact interaction searches; limits from the dijet resonance searches are weaker. Right panel: the same as on the left but now for $g_{A}^{t} / g_{A}^{q}=-1.5$. The two blue shaded regions correspond to a $90 \%$ and $95 \%$ CL fit to the data.

The reader will notice that the contact interaction search always provides a tighter constraint on our models than the dijet resonance search. This follows because the narrow resonance analyses only examine the shape difference without subtracting QCD background, while the contact interaction search has subtracted the QCD background and hence should constrain new physics more. Our constraints from dijet contact interactions are conservative because we neglected the NLO QCD correction. To have a more precise constraint, one should perform a NLO calculation as done in ref. [37].

\subsection{Phenomenological fit}

We first perform a model independent fit to $A_{F B}^{t \bar{t}}$ with one axigluon field, with a cross section given by eq. (2.1). In this case, there are five parameters that describe the axigluon contributions to $A_{F B}^{t \bar{t}}$ and the $t \bar{t}$ production cross section. Instead of scanning all of this parameter space, we set $g_{V}^{q}=g_{V}^{t}=0$ in this section and only consider three parameters: $M_{G^{\prime}}, g_{A}^{q}$ and $g_{A}^{t}$, which are most crucial for $A_{F B}^{t \bar{t}}$ when $M_{t \bar{t}} \ll M_{G^{\prime}}$. As discussed above, a negative value for the product $g_{A}^{q} g_{A}^{t}$ provides a positive contribution to $A_{F B}^{t \bar{t}}$. Therefore, we restrict the parameter space to $g_{A}^{q}>0$ and $g_{A}^{t}<0$ (the opposite choice would not change our discussion below).

In the contours displayed in figure 2 we fix the ratio $g_{A}^{t} / g_{A}^{q}$. In the left panel, we choose $g_{A}^{t}=-g_{A}^{q}$ and show the $90 \%$ and $95 \%$ C.L. fit with two shaded blue regions. The dark yellow solid line represents the constraint from dijet contact interaction searches. All parameter regions above and to the left of this line are excluded. The black dashed lines correspond to possible $20 \%$ and $40 \%$ axigluon contributions to the top quark pair 

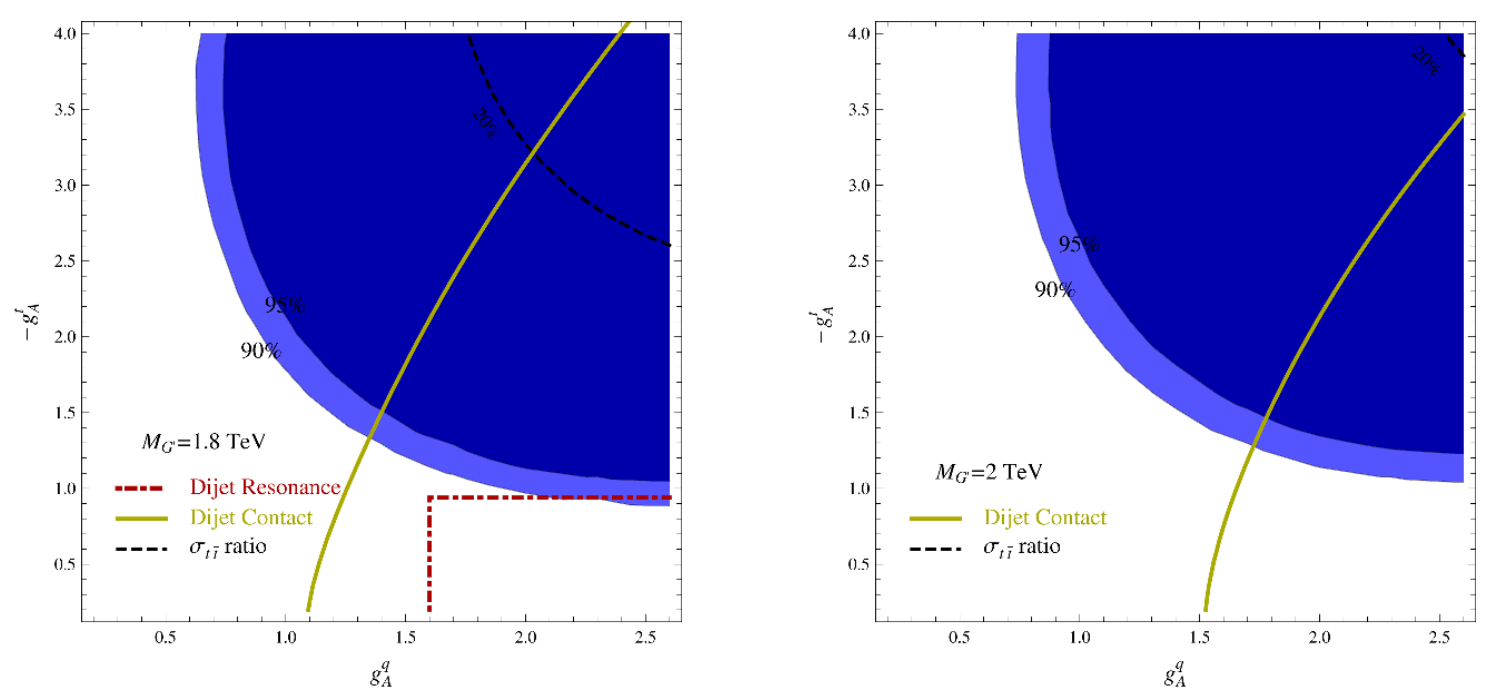

Figure 3. Left panel: the fit for the phenomenological model of eq. 2.1 with $g_{A}^{q}>0, g_{A}^{t}<0$ and $g_{V}^{q, t}=0$. The axigluon is assumed to have a mass of $M_{G^{\prime}}=1.8 \mathrm{TeV}$. The region on the right side of the dark yellow solid line is excluded by dijet contact interaction searches. Right panel: the same as on the left but now for $M_{G^{\prime}}=2 \mathrm{TeV}$. The narrow resonance dijet searches do not further constrain the parameter space.

production cross section, relative to that of the LO SM, for $M_{t \bar{t}}>450 \mathrm{GeV}$, as discussed in section 2.1. The upper limit for $g_{A}^{q}$ in this figure is chosen so that the $G^{\prime}$ width does not exceed its mass. The right panel in this figure is similar to the left but now for $g_{A}^{t}=-1.5 g_{A}^{q}$. As one can see from this figure, the low mass region is mostly ruled out by the dijet contact interaction searches, but a higher mass $G^{\prime}$ has plenty of parameter space. Concentrating on the high mass region, we next fix the $G^{\prime}$ mass and show the fit contour regions in figure 3 , where the left(right) panel is for $M_{G^{\prime}}=1.8(2.0) \mathrm{TeV}$. Comparing those two panels, one can see that more parameter space survives for a heavier $G^{\prime}$, which also has larger couplings and hence a larger width in the best-fit region. As an example, we show the fit results for $A_{F B}^{t \bar{t}}$ in table 2 with $M_{G^{\prime}}=2 \mathrm{TeV}, g_{A}^{q}=2.2, g_{A}^{t}=-3.2$ and $g_{V}^{q, t}=0$.

\section{Axigluon models}

In this section we will discuss three specific axigluon models that can be described in the language of dimensional deconstruction, or simply as coset models. The first and third are based on cosets $[\mathrm{SU}(3) \times \mathrm{SU}(3)] / \mathrm{SU}(3)_{c}$, while the second involves three $\mathrm{SU}(3)$ groups and two new color octet vector bosons. The third model differs from the first by the introduction of new vector-like quarks.

These models have elementary motivations. The first is the simplest possible coset model that produces an axigluon mediated $t \bar{t}$ forward-backward asymmetry, but we find it is excluded by the ATLAS dijet contact interaction search [36]. The second model improves on the first by allowing for heavier and more strongly coupled axigluons, yet we will see that 


\begin{tabular}{|c|c|c|}
\hline Selection & $M_{t \bar{t}}<450 \mathrm{GeV}$ & $M_{t \bar{t}}>450 \mathrm{GeV}$ \\
\hline Parton Level Exp. Data & $-0.116 \pm 0.146 \pm 0.047$ & $0.475 \pm 0.101 \pm 0.049$ \\
\hline Model Prediction & 0.10 & 0.31 \\
\hline Selection & $|\Delta y|<1.0$ & $|\Delta y|>1.0$ \\
\hline Parton Level Exp. Data & $0.026 \pm 0.104 \pm 0.056$ & $0.611 \pm 0.210 \pm 0.147$ \\
\hline Model Prediction & 0.12 & 0.40 \\
\hline
\end{tabular}

Table 2. The comparison of theoretic predictions and measured values for the phenomenological model with $M_{G^{\prime}}=2 \mathrm{TeV}, g_{A}^{q}=2.2, g_{A}^{t}=-3.2$ and $g_{V}=0$. The total $\chi^{2}$ is 5.5.

it is also excluded. The third model makes it possible to avoid the powerful dijet contact interaction search [36] by reducing the axigluon coupling to light quarks while increasing the axigluon coupling to $t \bar{t}$, thereby keeping the asymmetry fixed. Since constraints on $t \bar{t}$ production are weaker and less precise than the constraints on dijets, our third model survives - although it will be tested at the LHC in the coming months!

The models that follow provide calculable perturbative examples, but a more general theory such as our effective Lagrangian in section 2 might be the best new physics explanation for the $t \bar{t}$ asymmetry, in which case the asymmetry may be the first hint of new strongly coupled physics.

\subsection{The minimal axigluon model}

The simplest way to introduce another color octet vector boson is to extend the QCD gauge symmetry to the group $\mathrm{SU}(3)_{1} \times \mathrm{SU}(3)_{2}$, which then spontaneously breaks to its subgroup $\mathrm{SU}(3)_{c}$. This spontaneous symmetry breaking can be achieved by introducing a complex scalar field $\Sigma$ which transforms as a $(3, \overline{3})$ under the $\mathrm{SU}(3)_{1} \times \mathrm{SU}(3)_{2}$ symmetry with a non-zero VEV,$\langle\Sigma\rangle=\frac{\mathbb{I}_{3}}{\sqrt{6}} f_{\Sigma}$. There are only two parameters in this model and they can be identified as the axigluon mass $M_{G^{\prime}}$ and the gauge coupling mixing angle $\theta$. Here, we only consider $0 \leq \theta<45^{\circ}$, since the range $90^{\circ}-\theta$ can be mapped into the region $0 \leq \theta<45^{\circ}$ by flipping the quark charges under $\mathrm{SU}(3)_{1}$ and $\mathrm{SU}(3)_{2}$. Diagonalizing the mass matrix of the two gauge bosons $G_{1}^{\mu}$ and $G_{2}^{\mu}$, we obtain the massless QCD gluon,

$$
G^{\mu}=\cos \theta G_{1}^{\mu}+\sin \theta G_{2}^{\mu},
$$

and the massive axigluon state,

$$
G^{\prime \mu}=-\sin \theta G_{1}^{\mu}+\cos \theta G_{2}^{\mu} .
$$

The mixing angle $\theta$ is related to the gauge couplings of $\mathrm{SU}(3)_{1} \times \mathrm{SU}(3)_{2}, h_{1}$ and $h_{2}$ : $\tan \theta=h_{1} / h_{2}$. The QCD coupling is then given by $g_{s}=h_{1} \cos \theta=h_{2} \sin \theta$, and the mass of the axigluon is

$$
M_{G^{\prime}}=\frac{\sqrt{2} g_{s}}{\sqrt{3} \sin 2 \theta} f_{\Sigma}
$$


The other degrees of freedom in $\Sigma$ are assumed to be heavy for now, so we only have one new particle $G_{\mu}^{\prime}$ below the scale $\sim 4 \pi f_{\Sigma}$.

In order to obtain an $A_{F B}^{t \bar{t}}$ with the correct sign, we need opposite signs for the axialvector couplings of the axigluon to the light quarks and the top quark. To achieve this goal, we make the following assignments for the $\mathrm{SM}$ quarks under $\mathrm{SU}(3)_{1} \times \mathrm{SU}(3)_{2}: q_{L}, t_{R}, b_{R}$ as triplets of $\mathrm{SU}(3)_{1}$ and $(t, b)_{L}, q_{R}$ as triplets of $\mathrm{SU}(3)_{2}$ (see ref. [38] for a similar setup). To cancel the gauge anomalies, additional colored particles are required and are assumed to be heavy in here. Here, "q" represents the first two generations of quarks. With these charge assignments, we find the vector and axial-vector couplings of $G_{\mu}^{\prime}$ to the SM quarks, re-scaled by the QCD coupling $g_{s}$, to be

$$
g_{V}^{t}=g_{V}^{q}=\frac{1}{\tan 2 \theta}, \quad g_{A}^{t}=-g_{A}^{q}=\frac{1}{\sin 2 \theta} .
$$

Neglecting the quark masses (since $M_{G^{\prime}}$ will be at or above the TeV scale), the total decay width is found to be

$$
\Gamma\left(G^{\prime}\right)=\frac{\alpha_{s} M_{G^{\prime}}}{6}\left[4\left(\left|g_{V}^{q}\right|^{2}+\left|g_{A}^{q}\right|^{2}\right)+2\left(\left|g_{V}^{t}\right|^{2}+\left|g_{A}^{t}\right|^{2}\right)\right]=\alpha_{s} M_{G^{\prime}}\left(\frac{1}{\tan ^{2} 2 \theta}+\frac{1}{\sin ^{2} 2 \theta}\right) .
$$

For $\theta=\pi / 4$, one finds that the width to mass ratio of $G^{\prime}$ is $\Gamma\left(G^{\prime}\right) / M_{G^{\prime}}=\alpha_{s} \sim 10 \%$. We also note that if $G^{\prime}$ has other decay channels, it could become a broader resonance, a possibility we will consider later.

The interference term in $q \bar{q} \rightarrow t \bar{t}$ production between the $s$-channel gluon and $G^{\prime}$ contains a term linear in the cosine of the production angle of the $t$ in the $t \bar{t}$ rest frame; this leads to a forward-backward asymmetry (see eq. (2.1) and ref. [8]). We perform a fit to the observed and unfolded parton-level four-bin data from ref. [1], with the results being shown in figure 4, where the two blue contour regions correspond to the $90 \%$ and 95\% confidence level, respectively. In our fit, we have neglected the NLO SM contributions to $A_{F B}^{t \bar{t}}$ since we only include the leading order contribution from the new model; note that the radiative NLO SM asymmetry aligns with the asymmetry from the axigluon. The best-fit point is found to be at $M_{G^{\prime}}=1041 \mathrm{GeV}$ and $\theta=45^{\circ}$ with $\chi^{2} /$ d.o.f $=5.3 / 2$ for four bins, compared to $\chi^{2}=24.2 / 4$ for the SM tree-level only fit. The black dashed lines in figure 4, correspond to possible $20 \%$ and $40 \%$ axigluon contributions to the top quark pair production cross section, relative to that of the LO SM, for $M_{t \bar{t}}>450 \mathrm{GeV}$, as we discussed in section 2.1.

The axigluon couples to quark pairs with a coupling as strong as in QCD, therefore searches for possible dijet resonances [34] will further constrain the parameter space of the model, as discussed in section 2.2. We note that in the narrow width approximation the production cross section for $q \bar{q} \rightarrow G^{\prime} \rightarrow q \bar{q}$ is proportional to $\left|g_{V}^{q}\right|^{2}+\left|g_{A}^{q}\right|^{2}=\cot ^{2} 2 \theta+\csc ^{2} 2 \theta$ and that the branching fraction for $G^{\prime}$ decaying into jets is fixed to be $5 / 6$. Hence, as $\theta$ moves away from $45^{\circ}$, the production cross section increases and a stringent constraint can be obtained. Employing the CMS 95\% C.L. exclusion upper limit on the dijet resonance production $\sigma \times A$ from $q \bar{q}$ initial processes and then rescaling the cross sections for colorons and axigluons from figure 4 of ref. [34], we present the corresponding narrow dijet resonance 


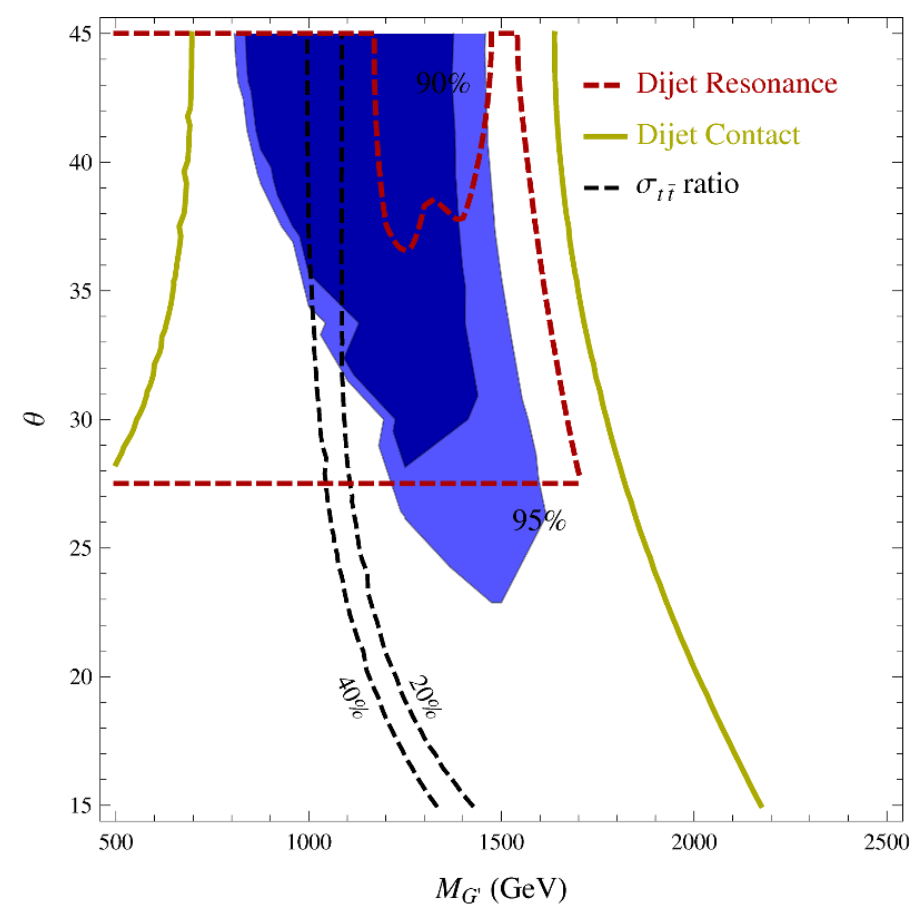

Figure 4. The fit of the minimal two-site axigluon model to the observed $A_{F B}^{t \bar{t}}$ at CDF. The four-bin values of $A_{F B}^{t \bar{t}}$ corresponding to high and low rapidity and high and low invariant mass are included in the $\chi^{2}$ analysis. The best fit point has a $\chi^{2} /$ d.o.f. $=5.3 / 2$ occurring at $M_{G^{\prime}}=1041 \mathrm{GeV}$ and $\theta=45^{\circ}$. The region enclosed by the red dashed lines is excluded by the dijet narrow resonance search at CMS [34] with $2.9 \mathrm{pb}^{-1}$ luminosity. The region between the dark solid yellow lines is excluded by the search for diquark contact interactions from ATLAS [36] with $3.1 \mathrm{pb}^{-1}$, so all of the preferred parameter space of this model has been eliminated.

constraints as the red dashed line in figure 4. Since these dijet resonance searches are based on the narrow width approximation, they will not constrain axigluons with large widths, as we saw with figure 1 .

Although a broad $G^{\prime}$ may survive the dijet resonance analyses, searches for quark contact interactions [36] via the dijet angular distributions can be used to constrain our model parameter space even when the $G^{\prime}$ is broad. The constraints from the dijet contact interaction searches are shown as the solid dark yellow lines of figure 4 . The parameter space enclosed by these two lines is excluded by this search. One can see that all of the $A_{F B}^{t \bar{t}}$ preferred parameter space regions are now ruled out. Therefore, we conclude that the minimal axigluon model can not adequately explain the $A_{F B}^{t \bar{t}}$ asymmetry observed at CDF.

One may wonder whether enlarging the width of the axigluon can help to evade these contact interaction constraints. There are many ways to make the axigluon broader by forcing the $G^{\prime}$ to decay into other modes. For instance, in ref. [39] the axigluon $G^{\prime}$ can decay into two scalar color-octets (if they are lighter than $G^{\prime}$ ), which belong to the other components of the $\Sigma$ field. The scalar octet may further decay into two jets, so there are then four jets in the final state and the direct dijet searches become much less of a 


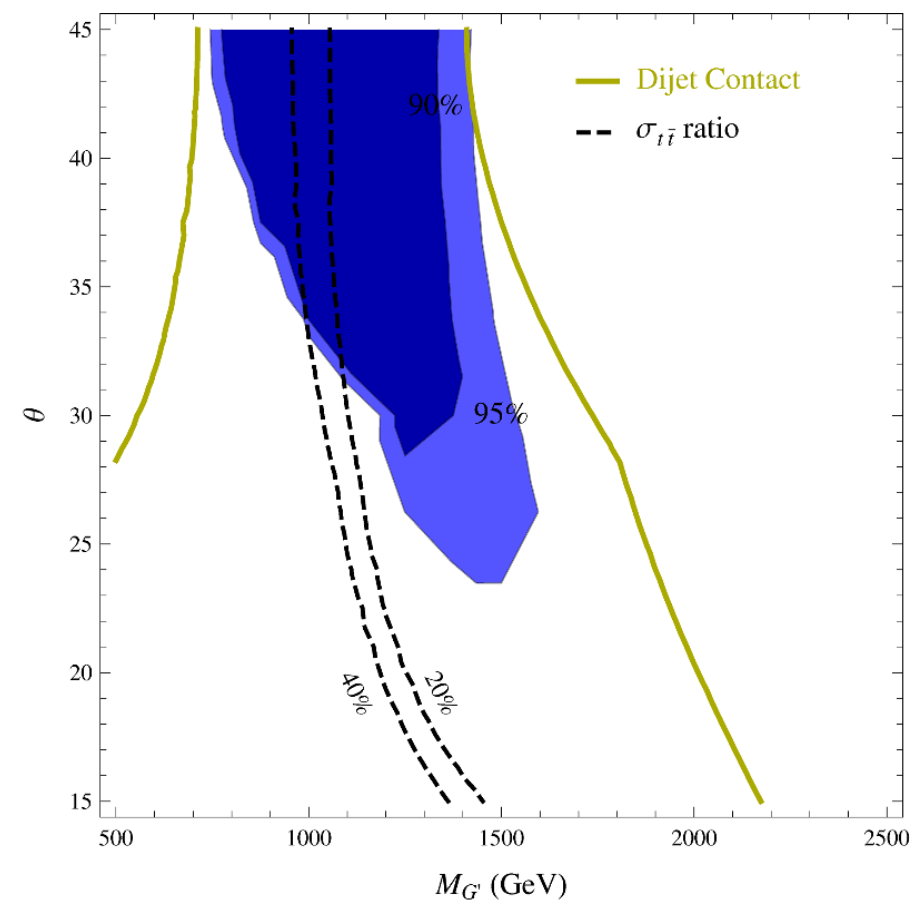

Figure 5. The same as figure 4 but for $\Gamma_{G^{\prime}} / M_{G^{\prime}} \geq 20 \%$. The dijet resonance searches no longer constrain this model.

constraint. Without specifying a specific model, we study the effects of changing the $G^{\prime}$ width on the resonant dijet searches in figure 5. In figure 5, we show the corresponding fitted results assuming the width/mass ratio of $G^{\prime}$ to be greater than $20 \%$ [if the width from eq. (3.5) is greater than $20 \%$ of the mass, we use the width in eq. (3.5)]. Although the narrow resonance searches do not apply in this case, the dijet contact interaction searches still exclude all of the $A_{F B}^{t \bar{t}}$ preferred parameter space region. Therefore, we must consider other extensions of the two-site axigluon model.

\subsection{A simplified three-site model}

One way to extend the minimal single axigluon model is to include an additional coloroctet gauge boson. In ref. [11], the authors compared the two-site model and the three-site model and concluded that the three-site model can not improve the fit to $A_{F B}^{t \bar{t}}$. Their arguments are based on the contact operator analysis obtained by integrating out heavy gauge bosons, which can be justified only when the heavy gauge bosons have narrow widths and have masses above the collider limit. However, one can see that the heavy gauge boson $G^{\prime}$ can be accessible at Tevatron from figure 4 and that the width can be fairly large, so it is worthwhile to briefly revisit the three site model.

In this section we will reconsider the three-site model with the gauge group $\mathrm{SU}(3)_{1} \times$ $\mathrm{SU}(3)_{2} \times \mathrm{SU}(3)_{3}$ plus two sigma fields $\Sigma_{1}$ and $\Sigma_{2}$, which transform as $(3, \overline{3})$ under $\mathrm{SU}(3)_{1} \times$ $\mathrm{SU}(3)_{2}$ and $(\overline{3}, 3)$ under $\mathrm{SU}(3)_{2} \times \mathrm{SU}(3)_{3}$. For simplicity, we will assume that the gauge 
couplings of $\mathrm{SU}(3)_{1}$ and $\mathrm{SU}(3)_{3}$ are identical and are given by $h_{1}$, with the corresponding coupling of $\mathrm{SU}(3)_{2}$ being $h_{2}$. We will further require that $\left\langle\Sigma_{1}\right\rangle=\left\langle\Sigma_{2}\right\rangle$, so there exists an $a d-h o c \mathbb{Z}_{2}$ symmetry in the gauge sector via the interchange of site "1" and site "3". Diagonalizing the gauge boson mass matrix, we determine the three mass eigenstates to be

$$
\begin{aligned}
G^{\mu} & =-\frac{\sin \theta}{\sqrt{2}} G_{1}^{\mu}+\cos \theta G_{2}^{\mu}-\frac{\sin \theta}{\sqrt{2}} G_{3}^{\mu}, \\
G^{\prime \mu} & =\frac{1}{\sqrt{2}} G_{1}^{\mu}-\frac{1}{\sqrt{2}} G_{3}^{\mu}, \\
G^{\prime \mu} & =\frac{\cos \theta}{\sqrt{2}} G_{1}^{\mu}+\sin \theta G_{2}^{\mu}+\frac{\cos \theta}{\sqrt{2}} G_{3}^{\mu} .
\end{aligned}
$$

Here, the mixing angle is defined as $\tan \theta \equiv \sqrt{2} h_{2} / h_{1}$ and the physical region is identified as $0 \leq \theta<90^{\circ}$. The mass ratio of the two massive gauge bosons is determined to be

$$
\frac{M_{G^{\prime \prime}}}{M_{G^{\prime}}}=\frac{1}{\cos \theta} .
$$

Assigning the quantum numbers to SM quarks: $q_{L}, t_{R}, b_{R}$ to be triplets of $\mathrm{SU}(3)_{1}$ and $(t, b)_{L}, q_{R}$ triplets of $\mathrm{SU}(3)_{3}$, we derive the couplings of $G_{\mu}^{\prime}$ to quarks to be (the $\mathbb{Z}_{2}$ symmetry is explicitly broken by the quark charge assignments, so that the $G_{\mu}^{\prime}$ is unstable)

$$
g_{V}^{t}=g_{V}^{q}=0, \quad g_{A}^{t}=-g_{A}^{q}=\frac{1}{\sin \theta},
$$

and the corresponding couplings of $G_{\mu}^{\prime \prime}$ to quarks are

$$
h_{V}^{t}=h_{V}^{q}=-\frac{1}{\tan \theta}, \quad h_{A}^{t}=-h_{A}^{q}=0 .
$$

Based on those couplings, we can see that $G^{\prime}$ behaves as an axigluon (with pure axial-vector couplings) and $G^{\prime \prime}$ as a coloron (with pure vector couplings).

Including the exchanges of all three gauge bosons in the $s$-channel, we find the differential production cross section to be given by

$$
\begin{aligned}
\frac{d \sigma^{q \bar{q} \rightarrow t \bar{t}}}{d \cos \theta^{*}}=\frac{\pi \alpha_{s}^{2} \beta}{9 \hat{s}} & \left\{1+c^{2}+4 m^{2}+\frac{2 \hat{s}\left(\hat{s}-M_{G^{\prime}}^{2}\right)}{\left(\hat{s}-M_{G^{\prime}}^{2}\right)^{2}+M_{G^{\prime}}^{2} \Gamma_{G^{\prime}}^{2}}\left(2 g_{A}^{q} g_{A}^{t} c\right)\right. \\
+ & \frac{2 \hat{s}\left(\hat{s}-M_{G^{\prime \prime}}^{2}\right)}{\left(\hat{s}-M_{G^{\prime \prime}}^{2}\right)^{2}+M_{G^{\prime \prime}}^{2} \Gamma_{G^{\prime \prime}}^{2}}\left[h_{V}^{q} h_{V}^{t}\left(1+c^{2}+4 m^{2}\right)\right] \\
& +\frac{\hat{s}^{2}}{\left(\hat{s}-M_{G^{\prime}}^{2}\right)^{2}+M_{G^{\prime}}^{2} \Gamma_{G^{\prime}}^{2}}\left[\left(g_{A}^{q} g_{A}^{t}\right)^{2}\left(1+c^{2}-4 m^{2}\right)\right] \\
& +\frac{\hat{s}^{2}}{\left(\hat{s}-M_{G^{\prime \prime}}^{2}\right)^{2}+M_{G^{\prime \prime}}^{2} \Gamma_{G^{\prime \prime}}^{2}}\left[\left(h_{V}^{q} h_{V}^{t}\right)^{2}\left(1+c^{2}+4 m^{2}\right)\right] \\
& \left.+\frac{\hat{s}^{2}\left[\left(\hat{s}-M_{G^{\prime}}^{2}\right)\left(\hat{s}-M_{G^{\prime \prime}}^{2}\right)+M_{G^{\prime}} M_{G^{\prime \prime}} \Gamma_{G^{\prime}} \Gamma_{G^{\prime \prime}}\right]}{\left[\left(\hat{s}-M_{G^{\prime}}^{2}\right)^{2}+M_{G^{\prime}}^{2} \Gamma_{G^{\prime}}^{2}\right]\left[\left(\hat{s}-M_{G^{\prime \prime}}^{2}\right)^{2}+M_{G^{\prime \prime}}^{2} \Gamma_{G^{\prime \prime}}^{2}\right]}\left(4 g_{A}^{q} g_{A}^{t} h_{V}^{q} h_{V}^{t} c\right)\right\}
\end{aligned}
$$

Here, $\beta=\sqrt{1-4 m^{2}}$ is the velocity of the top quark, with $m=m_{t} / \sqrt{\hat{s}}$, and $c=\beta \cos \theta^{*}$ with $\theta^{*}$ as the production angle of $t$ in the center of mass frame of $t \bar{t}$ system. 


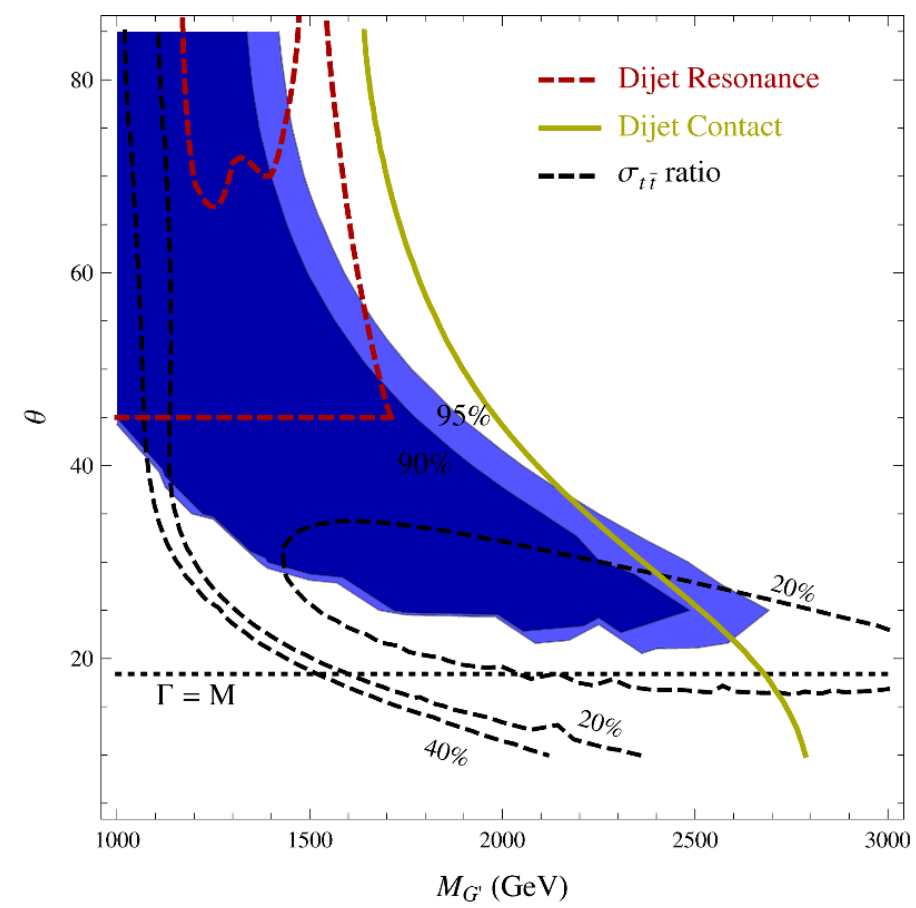

Figure 6. The fit of the simplified three-site model to the observed $A_{F B}^{t \bar{t}}$ at CDF. The best fit point has $\chi^{2} /$ d.o.f. $=4.4 / 2$ at $M_{G^{\prime}}=1.3 \mathrm{TeV}$ and $\theta=45^{\circ}$. Regions enclosed by the red dashed lines are excluded by the dijet narrow resonance searches. The region to the left side of the solid dark yellow line is ruled out by the search for dijet contact interactions. The $t \bar{t}$ production cross section itself does not further constrain the relevant parameter space.

Similar to the minimal two-site model, there are only two parameters in this model: $M_{G^{\prime}}$ and $\theta$. The corresponding $90 \%$ and $95 \%$ C.L. contour plot is shown in figure 6 . One can see from this figure that although there exists a large region that is not ruled out by the dijet narrow resonance searches, however the search for contact interactions in dijets indeed excludes almost all of the parameter space.

\subsection{A two-site model with a new vector-like quark}

All of the previous models were highly constrained by the dijet searches, especially by the search of dijet contact interactions. So in this section we consider a model which can relax the dijet constraints without reducing the top quark forward-backward asymmetry. We first notice that $A_{F B}^{t \bar{t}}$ only depends on the product of $g_{A}^{q}$ and $g_{A}^{t}$ for the two-site model while the dijet production cross section is only sensitive to the coupling $g_{A}^{q}$. If the relation between $g_{A}^{q}$ and $g_{A}^{t}$ as in eq. (3.4) of the minimal two-site model can be broken such that $g_{A}^{q}$ can be treated independently from $g_{A}^{t}$, one then has the freedom to relax the dijet constraints.

Returning to the gauge sector of the two-site model considered in section 3.1, we now add a new vector-like (under SM gauge group) fermion, $\psi_{L, R}$, which are $\mathrm{SU}(2)_{W}$ singlets and have charge $2 / 3$ under $\mathrm{U}(1)_{Y}$. Under the extended gauge group, we assign $q_{L}, q_{R}, t_{R}$, $b_{R}, \psi_{L}$ to be triplets of $\mathrm{SU}(3)_{1}$ and $(t, b)_{L}, \psi_{R}$ to be triplets of $\mathrm{SU}(3)_{2}$. In the up-type quark 
sector, the general $4 \times 4$ mass matrix can be diagonalized by a bi-unitary transformation acting on the left-handed and right-handed quarks. Since the left-handed mixing matrix is more highly constrained by the flavor observables, the right-handed mixing matrix has less serious constraints except for the mixing in the first two generations. Furthermore, because the first two-generation quarks have the same couplings under the new (flavor-changing) gauge boson $G^{\prime}$, the mixing between the right-handed quarks in the first two-generation is suppressed. To simplify our discussion, we assume the mixing matrix for the left-handed quarks to be approximately diagonal (we will discuss this assumption further in section 4) and introduce only one new mixing angle in the right-handed quark mixing matrix such that the new vector-like fermion only mixes with $u_{R}^{(f)}$ in the flavor basis.

The transition from the flavor basis to the mass eigenstate is then parametrized as

$$
\begin{aligned}
& u_{R}^{(m)}=\cos \alpha u_{R}^{(f)}+\sin \alpha \psi_{R}^{(f)}, \\
& \psi_{R}^{(m)}=-\sin \alpha u_{R}^{(f)}+\cos \alpha \psi_{R}^{(f)} .
\end{aligned}
$$

The couplings of the axigluon $G_{\mu}^{\prime}$ to the various quarks in the mass eigenstate basis are found to be

$$
\begin{aligned}
g_{V}^{(d, s, b, c)} & =-\tan \theta, & g_{A}^{(d, s, b, c)} & =0, \\
g_{V}^{t} & =\frac{1}{\tan 2 \theta}, & g_{A}^{t} & =\frac{1}{\sin 2 \theta}, \\
g_{V}^{u} & =-\tan \theta+\frac{\sin ^{2} \alpha}{\sin 2 \theta}, & g_{A}^{u} & =-\frac{\sin ^{2} \alpha}{\sin 2 \theta} .
\end{aligned}
$$

When $\alpha=\pi / 2$, the couplings of the up and top quarks become identical to those in the minimal two-site model. From the above equation, one can see that the axial-vector couplings of the up and top quarks are different and one now has the freedom to increase $g_{A}^{t}$ by reducing $\theta$ and to simultaneously decease $g_{V, A}^{u}$ at the same time by choosing $\sin ^{2} \alpha$ smaller than $\sin 2 \theta$. The total width of the $G_{\mu}^{\prime}$ in this model is then

$$
\Gamma\left(G^{\prime}\right)=\frac{\alpha_{s} M_{G^{\prime}}}{6} \frac{\left[\left(\cos ^{4} \alpha+10\right) \tan ^{2} \theta+\left(\sin ^{4} \alpha+1\right) \cot ^{2} \theta-2 \sin ^{2} \alpha \cos ^{2} \alpha\right]}{2} .
$$

For simplicity, we only introduce one vector-like fermion which mixes solely with the up quark. In principle, other vector-like fermions may also be present which mix with the charm and top quarks. Such a new quark that mixes with top can be assigned the same quantum numbers as the top, so that the $G^{\prime}$ coupling to the top quark remains unchanged. A new quark that mixes with charm can have the same quantum numbers as the new quark that mixes with the up quark. To evade potential flavor constraints, one can impose an $\mathrm{SU}(2)$ global symmetry, under which $\left(u_{R}, c_{R}\right)$ behave as an doublet and the two vector-like fermions as another doublet. For this choice, a mixing angle which is identical to $\alpha$ appears in the charm sector. The couplings $g_{A, V}^{c}$ will then be different from those in eq. (3.15) and the width of $G^{\prime}$ will be modifed; however, the general arguments in this section will be untouched.

Before we perform a general fit for this model to the $A_{F B}^{t \bar{t}}$ data, we note that there exists parameter space regions where there is no new physics contribution to the dominant 


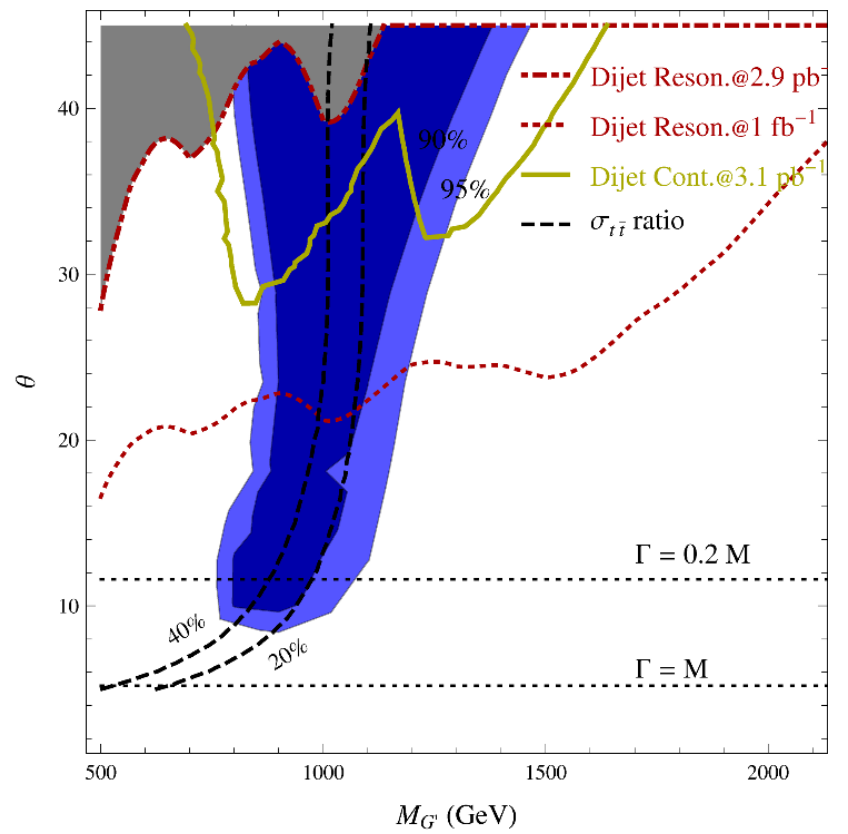

Figure 7. The fit for the model with one axigluon plus one additional vector-like fermion (section 3.3) to the observed $A_{F B}^{t \bar{t}}$ at CDF. The best fit point has $\chi^{2} /$ d.o.f. $=5.3 / 2$ at $M_{G^{\prime}}=1.05 \mathrm{TeV}$ and $\theta=45^{\circ}$. The region above the red dot-dashed line is excluded at 95\% C.L. by the dijet narrow resonance search, while the region above the dark yellow solid line is also excluded at 95\% C.L. by the search of dijet contact operator interactions. The projected exclusion limit from the dijet narrow resonance search at the $7 \mathrm{TeV} \mathrm{LHC}$ with $1 \mathrm{fb}^{-1}$ is shown by the red dotted line. The black dashed lines designate the regions (above and to the left) where the given percentage of the $t \bar{t}$ production cross section for $m_{t \bar{t}}>450 \mathrm{GeV}$ arises from new physics.

$t \bar{t}$ production process at the Tevatron: $u \bar{u} \rightarrow t \bar{t}$, to the leading order in $\hat{s} / M_{G^{\prime}}^{2}$. This can happen when $\sin \alpha=\sqrt{2} \sin \theta$ for $0 \leq \theta \leq \pi / 4$, which leads to $g_{V}^{u}=0$. Thus the contribution to the production cross section from the interference between the QCD gluon and the axigluon vanishes because it is proportional to the product $g_{V}^{u} g_{V}^{t}$. Let's first focus on this part of parameter space. The gauge couplings are then

$$
g_{V}^{(d, s, b, c)}=g_{A}^{u}=-\tan \theta, \quad g_{A}^{(d, s, b, c)}=g_{V}^{u}=0, \quad g_{V}^{t}=\frac{1}{\tan 2 \theta}, \quad g_{A}^{t}=\frac{1}{\sin 2 \theta} .
$$

The $G_{\mu}^{\prime}$ width is then simply

$$
\Gamma\left(G^{\prime}\right)=\frac{\alpha_{s} M_{G^{\prime}}}{6} \frac{\left(11 \tan ^{2} \theta+\cot ^{2} \theta\right)}{2} .
$$

The results of the fit are shown in figure 7, where the red dotdashed line is the constraint from the current dijet narrow resonance searches at CMS with $2.9 \mathrm{fb}^{-1}$, while the red dotted line is the projected exclusion limit for $1 \mathrm{fb}^{-1}$ at the LHC. The region above the dark yellow solid line is excluded by the search of dijet contact interactions from ATLAS with $3.1 \mathrm{pb}^{-1}$. Although there is no modification of the $t \bar{t}$ production cross section from 


\begin{tabular}{|c|c|c|}
\hline Selection & $M_{t \bar{t}}<450 \mathrm{GeV}$ & $M_{t \bar{t}}>450 \mathrm{GeV}$ \\
\hline Parton Level Exp. Data & $-0.116 \pm 0.146 \pm 0.047$ & $0.475 \pm 0.101 \pm 0.049$ \\
\hline Model Prediction & 0.06 & 0.21 \\
\hline Selection & $|\Delta y|<1.0$ & $|\Delta y|>1.0$ \\
\hline Parton Level Exp. Data & $0.026 \pm 0.104 \pm 0.056$ & $0.611 \pm 0.210 \pm 0.147$ \\
\hline Model Prediction & 0.08 & 0.27 \\
\hline
\end{tabular}

Table 3. The comparison of theoretic predictions and measured values for the two-site model with a new vector-like fermion. The $G^{\prime}$ mass is $1.1 \mathrm{TeV}$ and the mixing angle is $30^{\circ}$. The total $\chi^{2} /$ d.o.f is $8.3 / 2$.

the QCD and $G^{\prime}$ interference term, the $t \bar{t}$ production cross section is modified by the new-physics-only contribution and has the relative contribution with respect to the SM leading-order production cross section shown by the black dashed lines. From this figure, we conclude that in the two-site plus one vector-like fermion model there exist a small region of parameter space which is allowed by all of the constraints. As an example, we show the fit results of $A_{F B}^{t \bar{t}}$ in table 3 for $M_{G^{\prime}}=1.1 \mathrm{TeV}$ and $\theta=30^{\circ}$.

From the contours of figure 7, one can see that for a small mixing angle the best-fit region is insensitive to its exact value. This is easily understood since the product of the axial-vector couplings $g_{A}^{u} g_{A}^{t}=-1 /\left(2 \cos ^{2} \theta\right)$ is insensitive to $\theta$ for small $\theta$. In this figure, we also show two horizontal, dotted black lines with different width/mass ratios to show that in the best-fit region $G^{\prime}$ is a narrow resonance. One can see that a large fraction of the parameter space will be covered with increasing luminosity at the LHC.

For more general values of $\alpha$, we show the contour regions in figure 8 for $\alpha=45^{\circ}$ and $\alpha=30^{\circ}$, respectively. In those two figures, the QCD and $G^{\prime}$ interference term also contributes to the $t \bar{t}$ production cross section, so the parameter space is highly constrained by $t \bar{t}$ resonance searches. Comparing the two plots in figure 8 , we can see that for a smaller $\alpha$ a lighter $G^{\prime}$ is preferred by the fit to $A_{F B}^{t \bar{t}}$ because the product $g_{A}^{u} g_{A}^{t}$ is reduced. As a result, the parameter space for a smaller $\alpha$ leads to a larger $t \bar{t}$ production cross section and is thus less favored. For $\alpha=30^{\circ}$, the best fit is $\chi /$ d.o.f. $=2.6 / 2$ at $M_{G^{\prime}}=824 \mathrm{GeV}$ and $\theta=29^{\circ}$. For this point, we obtain $A_{F B}^{t \bar{t}}=(0.14,0.53)$ for the two different rapidity bins and $(0.06,0.42)$ for the two bins in invariant masses.

\section{Flavor constraints}

To reproduce the measured $t \bar{t}$ forward-backward asymmetry, the coupling of $G^{\prime}$ to the top quark is forced to have the opposite sign as the coupling of $G^{\prime}$ to the $u$ and $d$ quarks. This necessitates flavor violation at some level, because it means that the $G^{\prime}$ coupling cannot be proportional to the identity matrix in flavor space. Here, we compute the size of these flavor effects. 

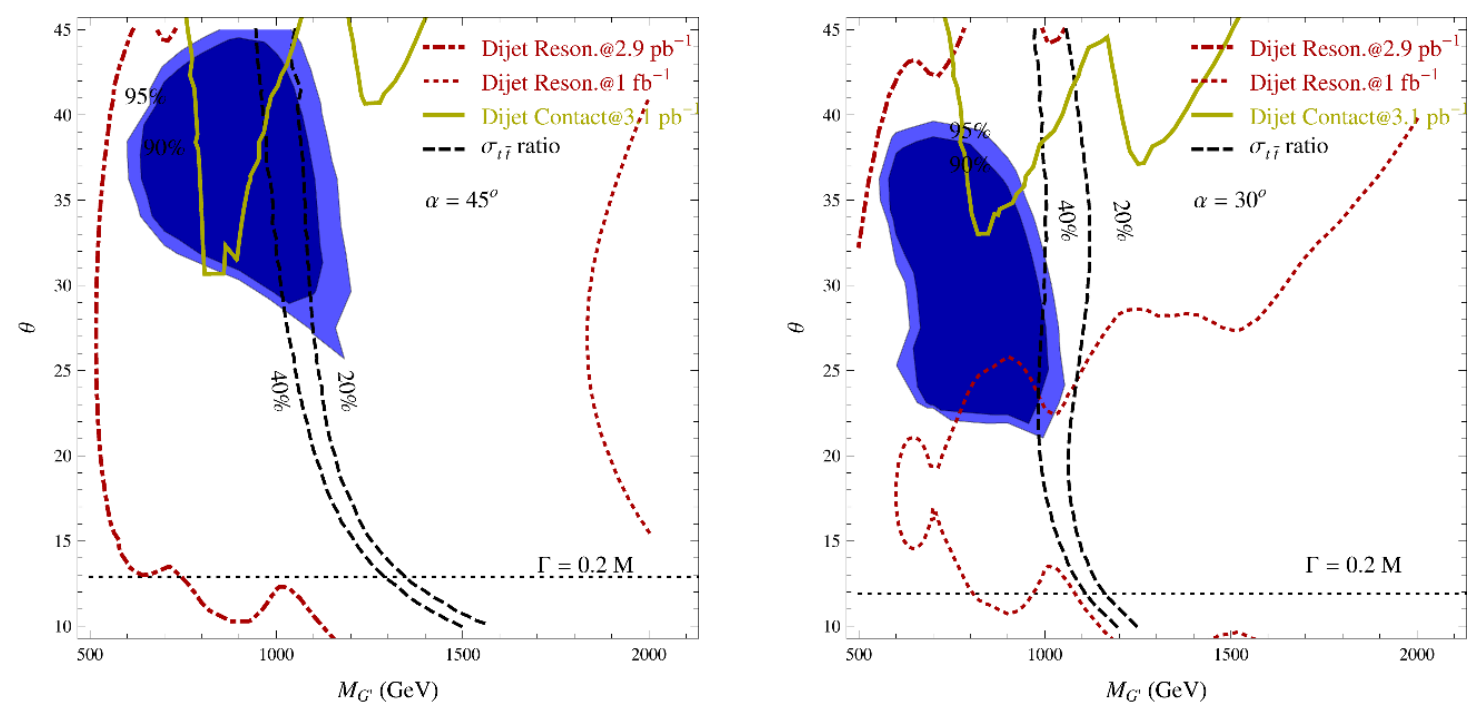

Figure 8. Left panel: the fit of the model with one axigluon plus one additional vector-like fermion (section 3.3) to the observed $A_{F B}^{t \bar{t}}$ at $\mathrm{CDF}$ with $\alpha=45^{\circ}$. The best fit point has $\chi^{2} /$ d.o.f. $=4.4 / 2$ at $M_{G^{\prime}}=849 \mathrm{GeV}$ and $\theta=42^{\circ}$. The region to the left of the red dotdashed line is excluded by the current dijet narrow resonance searches with luminosity $2.9 \mathrm{pb}^{-1}$. The region above the dark yellow solid line is excluded by the search for dijet contact interactions. Right panel: same as the left one but now for $\alpha=30^{\circ}$. The best fit point has a $\chi^{2} /$ d.o.f. $=2.6 / 2$ lying at $M_{G^{\prime}}=824 \mathrm{GeV}$ and $\theta=29^{\circ}$.

In order to determine the tree-level contributions of a heavy spin- 1 octet, $G^{\prime}$, to FCNC processes, we must first recall how they arise in a general theory [26]. Let $D^{0 T}=(d, s, b)^{0}$ and $U^{0 T}=(u, c, t)^{0}$ represent the three SM generations of $Q=-1 / 3$ and $Q=2 / 3$ quarks in the weak eigenstate basis, respectively. Spontaneous symmetry breaking via the Higgs Yukawa couplings then generates a mass matrix for these fields in the form $\bar{D}_{L}^{0} M^{d} D_{R}^{0}+$ h.c. (and similarly for the up-type quarks). In order to diagonalize this matrix, $M^{d}$, one needs to introduce the bi-unitary transformations $D_{L, R}=U_{L, R}^{D} D_{L, R}^{0}$ so that $U_{L}^{D} M^{d} U_{R}^{D \dagger}=M_{D}^{d}$ where $M_{D}^{d}$ is the $Q=-1 / 3$ diagonal mass matrix in the mass eigenstate basis. Note that if $M^{d}$ is Hermitian, we are then free to choose $U_{L}^{D}=U_{R}^{D}=U^{D}$. Note further that in this notation the conventional CKM matrix is given by a product of LH-rotations for the up-type and down-type quarks: $V_{C K M}=U_{L}^{U} U_{L}^{D \dagger}$ and that, in the SM, the RH-rotations play no role.

In what follows we will first compute the contribution of $G^{\prime}$ to $B_{q}-\bar{B}_{q}$ mixing (with $q=d, s)$, noting that the analysis for $D-\bar{D}$ mixing is identical with the substitutions $b \rightarrow c$ and $q \rightarrow u$. It is conceptually important to consider both types of mesons, because constraints from either alone might be evaded by pushing all the quark mixings into either the up or down-type sectors. We will also compute the constraints from $K-\bar{K}$ mixing via a direct matching to the operator coefficient limits given by the UTfit collaboration [40]. We use a different analysis for $K-\bar{K}$ because the strange quark is not heavy.

The interactions of the field $G^{\prime}$ with the $Q=-1 / 3$ fermions in the weak interaction 
basis are

$$
\mathcal{L}=-g_{s} \bar{D}_{L}^{0} A_{L} t^{a} \gamma^{\mu} D_{L}^{0} G_{\mu}^{\prime a}+(L \rightarrow R),
$$

where $t^{a}$ are the color generators and the matrices $A_{L(R)}=\operatorname{diag}\left(a_{1}, a_{2}, a_{3}\right)_{L(R)}$ are the explicit couplings in this basis. Note that the $A$ matrices are bi-fundamentals under SU(3) flavor symmetries, and as such, they are a new source of flavor violation. Rotating to the mass eigenstate basis, we have new couplings that take the explicit form

$$
C_{L, k j}^{D}=\left(U_{L}^{D}\right)_{k m} a_{L m} \delta_{m i}\left(U_{L}^{D \dagger}\right)_{i j},
$$

and similarly for $L \rightarrow R$. Here, we are specifically interested in the case of off-diagonal couplings, $k \neq j$ with $\left(a_{1}=a_{2} \neq a_{3}\right)_{L(R)}$ as we are assuming that the first two generations have identical couplings. Since by unitarity $\left(U_{L}^{D}\right)_{k i}\left(U_{L}^{D \dagger}\right)_{i j}=\delta_{k j}$, and similarly for the right-handed and up-type sectors, we find the off-diagonal couplings to be given by

$$
C_{L, k j}^{D}=\left(a_{3}-a_{1}\right)_{L}\left(U_{L}^{D}\right)_{k 3}\left(U_{L}^{D \dagger}\right)_{3 j}=\left(a_{3}-a_{1}\right)_{L}\left(U_{L}^{D}\right)_{k 3}\left(U_{L}^{D *}\right)_{j 3} \quad k \neq j,
$$

and similarly for $L \rightarrow R$. Of course, all of this naturally repeats itself in the $Q=2 / 3$ sector, with $U_{L}^{D} \rightarrow U_{L}^{U}$.

Now consider the FCNC contributions from $G^{\prime}$ to $B_{q^{-}} \bar{B}_{q}$ mixing; the effective Lagrangian is

$$
\mathcal{L}_{\mathrm{FCNC}}=-g_{s} \bar{b} t^{a} \gamma^{\mu}\left(C_{L, 3 q}^{D} P_{L}+C_{R, 3 q}^{D} P_{R}\right) q G_{a}^{\prime \mu}+\text { h.c. } .
$$

To obtain our results we follow the discussion as given in section 4.2 of ref. [41] which contains a very similar set-up. The procedure is, essentially, as follows: $(i)$ draw the contributing diagrams arising from $\mathcal{L}_{\mathrm{FCNC}}$ and integrate $G^{\prime}$ out. (ii) Contract the color structure and Fierz transform the product of the two currents to express the resulting expression in terms of a known basis of 4 -fermion operators [42]. (iii) Compute the renormalization group running of these operators down to the scale of the meson masses, and compute the appropriate matrix elements. Following ref. [41], the effective Hamiltonian is given by

$$
\mathcal{H}_{\Delta B=2}=\frac{2 \pi \alpha_{s}}{3 M_{G^{\prime}}^{2}}\left[\left(C_{L, 3 q}^{D}\right)^{2} Q_{1 L}+\left(C_{R, 3 q}^{D}\right)^{2} Q_{1 R}-C_{L, 3 q}^{D} C_{R}^{D} Q_{2}-6 C_{L, 3 q}^{D} C_{R, 3 q}^{D} Q_{3}\right],
$$

with the operators

$$
\begin{aligned}
Q_{1 L, R} & =\left(\bar{q}_{L, R} \gamma_{\mu} b_{L, R}\right)\left(\bar{q}_{L, R} \gamma^{\mu} b_{L, R}\right), \\
Q_{2} & =\left(\bar{q}_{L} \gamma_{\mu} b_{L}\right)\left(\bar{q}_{R} \gamma_{\mu} b_{R}\right), \\
Q_{3} & =\left(\bar{q}_{L} b_{R}\right)\left(\bar{q}_{R} b_{L}\right) .
\end{aligned}
$$

This Hamiltonian describes $B, D$, and $K$ meson mixing with appropriate replacements.

To proceed in the case of $B-\bar{B}$ mixing, we compute the matrix elements of $\mathcal{H}_{\Delta B=2}$ and compare to bounds from the UTfit Collaboration [40]. We present the results in terms of the matrix element of the LL operator $Q_{1 L}$, which we write as $r_{1}\left\langle Q_{1 L}\right\rangle$, where $r_{1} \simeq 0.77$ and accounts for the renormalization group running of $Q_{1 L}$ from the $\mathrm{TeV}$ scale to the $b$ 
quark mass, as is appropriate for $B-\bar{B}$ mixing $\left(r_{1} \simeq 0.70\right.$ for $D-\bar{D}$ mixing and accounts for the additional RG evolution to the charm quark mass). The matrix elements of the other operators differ by factors that we write as $R_{2,3}$, which account for their RG running, so in total we find

$$
\left\langle B\left|\mathcal{H}_{\Delta B=2}\right| \bar{B}\right\rangle=\frac{2 \pi \alpha_{s}}{3 M_{G^{\prime}}^{2}}\left[\left(C_{L, 3 q}^{D}\right)^{2}+\left(C_{R, 3 q}^{D}\right)^{2}-C_{L, 3 q}^{D} C_{R, 3 q}^{D} R_{2}-6 C_{L, 3 q}^{D} C_{R, 3 q}^{D} R_{3}\right],
$$

with $R_{2,3}$ given by the expressions in [42] such that (taking $N_{c}=3$ )

$$
R_{2}=\frac{1}{\sqrt{r}_{1}}\left(-\frac{3}{4}-\frac{1}{2} \frac{M_{B}^{2}}{\left(m_{b}+m_{q}\right)^{2}}\right), \quad R_{3}=\frac{1}{r_{1}^{5}}\left(\frac{1}{8}+\frac{3}{4} \frac{M_{B}^{2}}{\left(m_{b}+m_{q}\right)^{2}}\right) .
$$

Numerically we obtain $R_{2} \simeq-1.7$ and $R_{3} \simeq 4$.8. The bound on $M_{G^{\prime}}$ is then obtained by comparing to the cutoff limit from UTfit [40]:

$$
\frac{2 \pi \alpha_{s}}{3 M_{G^{\prime}}^{2}}\left|\left(C_{L, 3 q}^{D}\right)^{2}+\left(C_{R, 3 q}^{D}\right)^{2}-27 C_{L, 3 q}^{D} C_{R, 3 q}^{D}\right|<\frac{1}{\Lambda_{B}^{2}},
$$

with $\Lambda_{B}=210(30) \mathrm{TeV}$ for $B_{d}\left(B_{s}\right)$ mixing. This also allows a direct comparison to the analysis of ref. [11], with which we disagree. Numerically this reduces, in the case of $B_{d}$ mixing, to

$$
M_{G^{\prime}} \gtrsim(100 \mathrm{TeV})\left|\left(C_{L, 31}^{D}\right)^{2}+\left(C_{R, 31}^{D}\right)^{2}-27 C_{L, 31}^{D} C_{R, 31}^{D}\right|^{1 / 2} .
$$

An identical analysis applied to the up-type sector for $D-\bar{D}$ mixing gives

$$
\frac{2 \pi \alpha_{s}}{3 M_{G^{\prime}}^{2}}\left|\left(C_{L, 21}^{U}\right)^{2}+\left(C_{R, 21}^{U}\right)^{2}-60 C_{L, 21}^{U} C_{R, 21}^{U}\right|^{2}<\frac{1}{\Lambda_{D}^{2}},
$$

and numerically with $\Lambda_{D}=1.2 \times 10^{3} \mathrm{TeV}$ [40] we find

$$
M_{G^{\prime}} \gtrsim(600 \mathrm{TeV})\left|\left(C_{L, 21}^{U}\right)^{2}+\left(C_{R, 21}^{U}\right)^{2}-60 C_{L, 21}^{U} C_{R, 21}^{U}\right|^{1 / 2} .
$$

In the case of $K-\bar{K}$ mixing we will directly compare the coefficients of these operators to the UTfit bounds [40]. From $Q_{1 L, R}$ we find that

$$
M_{G^{\prime}} \gtrsim(500 \mathrm{TeV})\left|\left(C_{L, 21}^{D}\right)^{2}+\left(C_{R, 21}^{D}\right)^{2}\right|^{1 / 2},
$$

and from $Q_{3}$

$$
M_{G^{\prime}} \gtrsim(5000 \mathrm{TeV})\left|C_{L, 21}^{D} C_{R, 21}^{D}\right|^{1 / 2} .
$$

These limits derive from the real part of the $K-\bar{K}$ mixing; the imaginary part gives limits that are stronger by a factor of 15 .

We cannot proceed without making model-dependent assumptions, so in particular, we cannot set a firm exclusion limit. To give a reasonable picture of the situation, we will consider two very simple model assumptions, where $(\alpha)$ the quark mass matrices are Hermitian so that $U_{L}^{D}=U_{R}^{D}=U^{D}$ and $U_{L}^{U}=U_{R}^{U}=U^{U}$ and $(\beta)$ where the right handed $U_{R}^{U}=U_{R}^{D}=\mathbb{I}_{3}$. Furthermore, for convenience we assume that all three generations have identical vector couplings to $G^{\prime}$, while the first two generations and the third have distinct axial couplings. 


\begin{tabular}{|c|c|c|}
\hline & \multicolumn{2}{|c|}{ Lower bound on $M_{G^{\prime}}$ in TeV } \\
\hline & $(\alpha)$ & $(\beta)$ \\
\hline Kaon System & $5000 \times\left|U_{23}^{D} U_{13}^{D *}\right|$ & $500 \times\left|U_{23}^{D} U_{13}^{D *}\right|$ \\
\hline$B$ Mixing & $500 \times\left|U_{33}^{D} U_{13}^{D *}\right|$ & $100 \times\left|U_{33}^{D} U_{13}^{D *}\right|$ \\
\hline$D \quad$ Mixing & $4500 \times\left|U_{23}^{U} U_{13}^{U *}\right|$ & $600 \times\left|U_{23}^{U} U_{13}^{U *}\right|$ \\
\hline
\end{tabular}

Table 4. The constraints on the axigluon mass and mixing matrices from meson mixing for the two model choices discussed in the text. The common multiplicative factor $\left|g_{A}^{t}-g_{A}^{q}\right|$ is not included in the table. The Kaon limit would be a factor of 15 stronger in the presence of $O(1)$ phases.

For the choice $(\alpha)$ we obtain (with the couplings defined in units of $g_{s}$ )

$$
C_{L, 31}^{D}=-C_{R, 31}^{D}=\left(g_{A}^{t}-g_{A}^{q}\right) U_{33}^{D} U_{13}^{D *} \text { and } C_{L, 21}^{U}=-C_{R, 21}^{U}=\left(g_{A}^{t}-g_{A}^{q}\right) U_{23}^{U} U_{13}^{U *},
$$

note that because $G^{\prime}$ couples universally to the first two generations, to induce an FCNC we must couple through the third generation. The numerical bounds are listed in the second column of table 4. Alternatively, for the choice $(\beta)$ we have

$$
C_{L, k j}^{U, D}=\left(g_{A}^{t}-g_{A}^{q}\right) U_{k 3}^{U, D} U_{j 3}^{U, D *} \quad \text { and } \quad C_{R, k j}^{D}=-C_{R, k j}^{U}=0
$$

and so we avoid the large RG enhancement, giving the weaker constraints listed in the third column of table 4 .

Although our models determine the coupling $g_{A}^{t, q}$, without an accompanying model of flavor we cannot determine what values the matrix elements of $U$ take. All that we know are the elements of the CKM matrix, $V_{C K M}=U_{L}^{U} U_{L}^{D \dagger}$. In the general case all elements of the matrices $U_{L, R}^{U, D}$ could enter the observables, so we can no longer operate in a basis where, e.g., one of the original mass matrices is diagonal. If we assume (without any particular justification) that $\left|U_{33}^{D} U_{13}^{D *}\right|=\left|V_{t d}^{*} V_{t b}\right| \simeq 8.4 \cdot 10^{-3}$ and $\left|U_{23}^{D} U_{13}^{D *}\right|=\left|V_{t s}^{*} V_{t d}\right| \simeq 3.5 \cdot 10^{-4}$ [43] then we find that $M_{G^{\prime}} \gtrsim 5\left|g_{A}^{t}-g_{A}^{q}\right| \mathrm{TeV}$ for the choice $(\alpha)$, and $M_{G^{\prime}} \gtrsim 0.8\left|g_{A}^{t}-g_{A}^{q}\right| \mathrm{TeV}$ for choice $(\beta)$. Here we have assumed that all complex phases are small; if we assume $\mathcal{O}(1)$ phases then these constraints become about a factor of three stronger. Note that in the extreme limit that $U_{L}^{D}=\mathbb{I}_{3}$ while $V_{C K M}=U_{L}^{U}, M_{G^{\prime}}$ and $g_{A}$ are completely unconstrained by considerations of flavor in the down sector. Then the only constraint comes from $D-\bar{D}$ mixing, and we have $\left|U_{23}^{U} U_{13}^{U *}\right|=\left|V_{t s}^{*} V_{t d}\right| \simeq 3.5 \cdot 10^{-4}$, giving $M_{G^{\prime}} \gtrsim 1.6\left|g_{A}^{t}-g_{A}^{q}\right| \mathrm{TeV}$ for $(\alpha)$, and $M_{G^{\prime}} \gtrsim 200\left|g_{A}^{t}-g_{A}^{q}\right| \mathrm{GeV}$ for choice $(\beta)$. The latter constraint is trivially satisfied by all of our models.

To summarize: there are no model independent flavor constraints; generic axigluon models have significant tension with flavor, but there are plausible flavor scenarios where this tension disappears. 


\section{Signatures at the LHC and Tevatron}

The axigluon explanation of the observed $t \bar{t}$ forward-backward asymmetry can be tested at the LHC in either the dijet or the $t \bar{t}$ final state. Almost all of the $A_{F B}^{t \bar{t}}$ preferred parameter space in the above models will be explored with $1 \mathrm{fb}^{-1}$ of data at the 7 or $8 \mathrm{TeV}$ LHC.

\subsection{Dijet resonances}

By rescaling the results of the CMS dijet narrow resonance search [34] performed with 2.9 $\mathrm{pb}^{-1}$, we can determine how much luminosity will be needed to discover a given model. For instance, for the model with one axigluon and one vectorlike fermion presented above with $M_{G^{\prime}}=1.1 \mathrm{TeV}$ and a mixing angle of $\theta=30^{\circ}$, the dijet production cross section is suppressed by $1 / 5$ relative to a standard "coloron" model [17], so naively we expect that about $35 \mathrm{pb}^{-1}$ will be sufficient to exclude this model point at $95 \%$ C.L. On the other hand, with a luminosity $\sim 250 \mathrm{pb}^{-1}$ the axigluon with this set of parameters will be discovered at the $7 \mathrm{TeV}$ LHC. The projected exclusion limits with $1 \mathrm{fb}^{-1}$ of luminosity is presented in figure 7 . We see that if it exists, a new axigluon resonance that can explain the $t \bar{t}$ forward-backward asymmetry should be discovered sometime this year!

For an axigluon described by the phenomenological model presented in figure 3 , its width should be greater than $20 \%$ of its mass and it will appear as a wide resonance. The dijet narrow resonance searches will likely no longer be applicable. One could then employ an analysis similar to the quark contact interaction searches [36] using the dijet rapidity difference to distinguish the signal events from the background. For $M_{G^{\prime}}=2 \mathrm{TeV}$, $g_{A}^{u}=1.5, g_{A}^{t}=-2$ and $g_{V}=0$, one can exclude this axigluon at $95 \%$ C.L. with $\sim 44 \mathrm{pb}^{-1}$ of data assuming a $5 \%$ systematic error on the SM backgrounds following the analysis presented in [36]. However, in order to obtain a $5 \sigma$ discovery, one needs to modify the strategy appearing in [36] by choosing appropriate invariant mass bins and comparing the signal and background shapes in order to reduce this systematic error. We encourage the ATLAS and CMS collaborations to adapt their dijet searches in order to cover all the parameter space of the above phenomenological model.

\subsection{Top-anti-top resonances}

As discussed above, the $t \bar{t}$ mass distribution is quite sensitive to the presence of a new color-octet vector boson. The large $t \bar{t}$ cross section [44] coupled with the increased mass reach make the LHC an ideal environment for such resonance searches. Here, we explore the reach of the LHC in this observable for some $A_{F B}^{t \bar{t}}$-preferred and viable parameter points of the above axigluon models.

The new axigluon, which does not couple to two gluons, is exchanged only in $s$-channel $q \bar{q}$ annihilation and so does not interfere with the gluon fusion contribution. The signature is striking as shown in figure 9 . Here, the red line displays the $t \bar{t}$ invariant mass distribution at the $8 \mathrm{TeV}$ LHC for the model with one axigluon and one vectorlike fermion discussed above, taking $M_{G^{\prime}}=1100 \mathrm{GeV}$ and $\theta=30^{\circ}$, while the black curve represents the SM. With $1 \mathrm{fb}^{-1}$ luminosity, it is clear that one can easily find this new resonance on top of the SM background. The green (blue) line shows the contributions arising from the SM plus the 


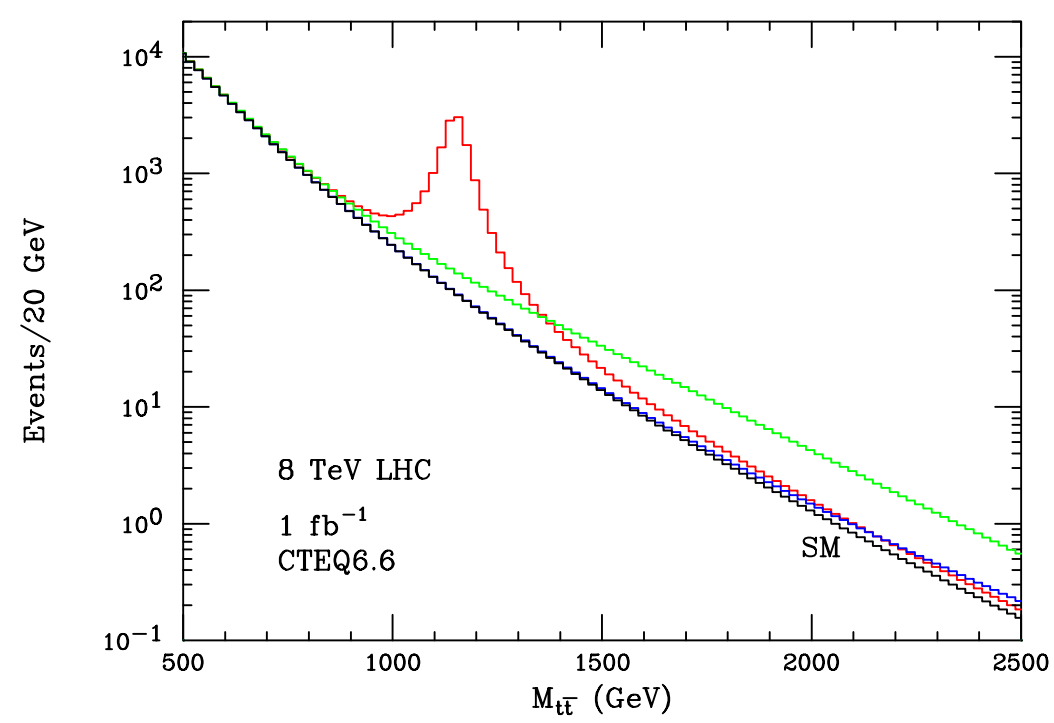

Figure 9. The $M_{t \bar{t}}$ distribution from the axigluon contribution plus the SM background at leading order. The red line is for the model with one axigluon and one vectorlike fermion described above with $M_{G^{\prime}}=1100 \mathrm{GeV}$ and $\theta=30^{\circ}$. The green(blue) line is for the phenomenological axigluon model with $M_{G^{\prime}}=1(2) \mathrm{TeV}, g_{A}^{u}=1.5, g_{A}^{t}=-2$ and $g_{V}=0$. The black line represents the SM.

phenomenological axigluon model with $M_{G^{\prime}}=1(2) \mathrm{TeV}, g_{A}^{u}=1.5, g_{A}^{t}=-2$ and $g_{V}=0$. Since $\Gamma / M \approx 28 \%$, no obvious bump appears in the distribution and one needs to employ a contact interaction search in $t \bar{t}$ final states.

We next estimate the sensitivity of the $8 \mathrm{TeV}$ LHC to the presence of these new states. We model our analysis after the strategy detailed by the ATLAS Collaboration for generic $t \bar{t}$ resonance searches $[45,46]$, including a new color octet boson from technicolor models. We only consider the semi-leptonic top-quark decays into muons and electrons (see ref. [47-49] for detailed studies with the $14 \mathrm{TeV}$ LHC). Other channels, such as the dilepton channel [50] can also be used to improve the discovery sensitivity. The ATLAS studies find that the main source of background for $t \bar{t}$ resonances originates from SM top pair production, while reducible backgrounds such as $W+$ jets are negligible for resonance searches. The resulting dominant background arises from combinatorics. We employ the $M_{t \bar{t}}$ reconstruction efficiency presented in [45, 46], which is at the level of a few percent and drops significantly for increasing $M_{t \bar{t}}$. We note that new techniques have recently been developed that reconstruct top-quarks produced with large enough transverse momentum such that their decay products can be tagged as a single jet [51-54]; however employing these methods is beyond the scope of our simple analysis here. We estimate the combined uncertainties in the NLO $t \bar{t}$ cross section and the parton distribution functions at high invariant masses to be of order $50 \%[55,56]$.

Requiring $S / \sqrt{B} \geq 5$ as a discovery criteria, and including both statistical and $50 \%$ systematic theoretical errors, we find that for the model with one axigluon and one vec- 
torlike fermion and taking $\theta=30^{\circ}$, the axigluon can be discovered up to $1.7 \mathrm{TeV}$ at the $8 \mathrm{TeV}$ LHC with $1 \mathrm{fb}^{-1}$. As the parameter $\theta$ is varied, we find that the axigluon cannot be observed at the level of $S / \sqrt{B} \geq 5$ for $\theta<13^{\circ}$. For $\theta \geq 13^{\circ}$, the axigluon search reach is in the range $1100-1700 \mathrm{GeV}$ as $\theta$ increases in value. Thus, the $A_{F B}^{t \bar{t}}$-preferred region in figure 7 with a narrow axigluon or at large $\theta$ will be probed by the early LHC data. For the phenomenological axigluon model with a large axigluon width presented in figure 3 , a narrow resonance search is no longer applicable and a contact interaction analysis with $t \bar{t}$ final states must be performed. However, it is clear from figure 9 large luminosities or an increase in center-of-mass energy will be required to observe a $\sim 2 \mathrm{TeV}$ axigluon in the $t \bar{t}$ channel for this scenario. For such heavy states, their contribution to $M_{t \bar{t}}$ is of order the NLO and PDF uncertainties.

\subsection{Production in association with a weak gauge boson}

Another signature for axigluons at the LHC is to study $G^{\prime}$ production together with a weak gauge boson. For the production of $p p \rightarrow G^{\prime}+W^{ \pm}$, the final state is $2 j+\ell+\mathbb{E}_{T}$, which has a smaller background than the dijet resonance channel and thus may have a better discovery reach at the LHC. The partonic production cross section in leading order QCD is calculated to be [39]

$$
\begin{aligned}
\sigma\left(u \bar{d} \rightarrow G_{\mu}^{\prime} W^{+}\right)=\frac{8 \pi \alpha \alpha_{s}\left(\left|g_{V}^{q}\right|^{2}+\left|g_{A}^{q}\right|^{2}\right)}{9 \hat{s}^{2} \sin ^{2} \theta_{W}} \times \\
\times\left[\frac{\hat{s}^{2}+\left(M_{G^{\prime}}^{2}+M_{W}^{2}\right)^{2}}{\hat{s}-M_{G^{\prime}}^{2}-M_{W}^{2}} \ln \left(\frac{\hat{s}-M_{G^{\prime}}^{2}-M_{W}^{2}+M_{\beta}^{2}}{2 M_{G^{\prime}} M_{W}}\right)-M_{\beta}^{2}\right],
\end{aligned}
$$

where

$$
M_{\beta}^{2}=\left[\left(\hat{s}-M_{G^{\prime}}^{2}-M_{W}^{2}\right)^{2}-4 M_{G^{\prime}}^{2} M_{W}^{2}\right]^{1 / 2} .
$$

Convoluting this partonic cross section with the MSTW [57] PDFs, we have the production cross sections at the LHC shown in figure 10. The production cross section of $G^{\prime}+W^{-}$ is smaller than $G^{\prime}+W^{+}$and is not shown in this figure. The major background comes from $W^{+}$plus jets from QCD and the discovery limit requires a detailed analysis, which is beyond the scope of this paper.

\subsection{Bottom quark forward-backward asymmetry}

An anomalously large forward-backward asymmetry in $t \bar{t}$ suggests the possibility of a similar asymmetry in $b \bar{b}$. Models that preserve a custodial $\mathrm{SU}(2)_{R}$ symmetry give rise to asymmetries of roughly equal magnitude in $t \bar{t}$ and $b \bar{b}$, so a measurement of the forwardbackward asymmetry in $b$ quarks would provide further insight into the nature of possible new physics contributions [4].

Let us explore the feasibility of a $b \bar{b}$ asymmetry measurement using the top quark data as a guide. Since the $t \bar{t}$ asymmetry was only significant for $M_{t \bar{t}}>450 \mathrm{GeV}$ and $\Delta y>1$, it is natural to assume that an asymmetry in $b$ quarks will be most visible in an analogous region. Furthermore, in order to tag the sign of a $b$ quark, the $B$ meson must decay to a visible electron or a muon. At CDF this requires $|y|<1$ for any tagged $b$-jet, and in any 


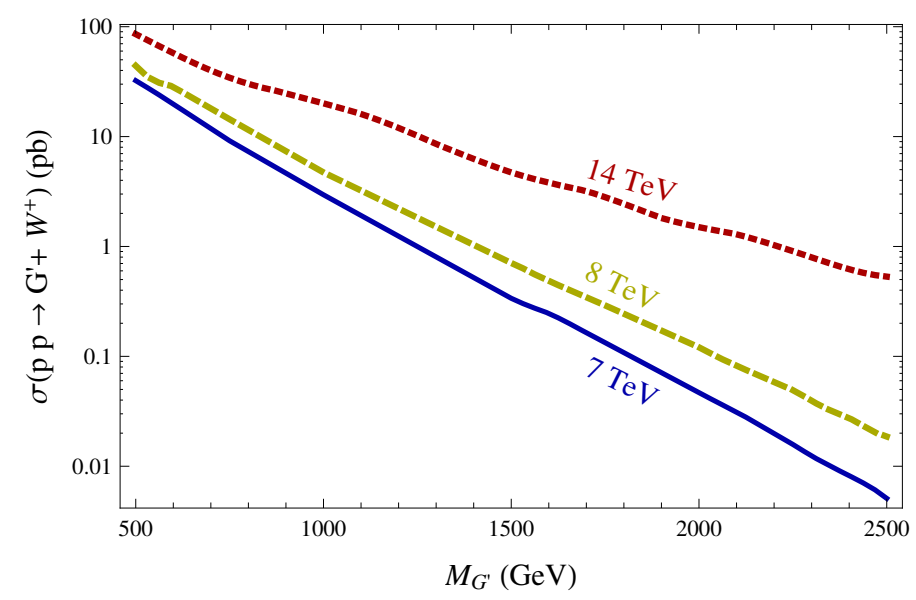

Figure 10. The production cross section of $G^{\prime}$ in association with $W^{+}$at the LHC for three different center of mass energies. The couplings of $G^{\prime}$ to light quarks are chosen to be $\left|g_{V}^{q}\right|^{2}+\left|g_{A}^{q}\right|^{2}=2$.

case $b$-tagging itself quickly loses efficiency outside of this central region [58]. ${ }^{3}$ To estimate the number of events available for a $b \bar{b}$ asymmetry measurement with these rapidity and invariant mass constraints, we integrated MSTW PDFs [57] using the leading order QCD cross section, resulting in a cross section times acceptance of $0.3 \mathrm{pb}$ for $M_{b \bar{b}}>450 \mathrm{GeV}$. So with a full data set of $10 \mathrm{fb}^{-1}$, there would be about $3000 b \bar{b}$ events to work with. However, it is possible that the $t \bar{t}$ asymmetry was dominated by the region $\Delta y>1$ in part because for smaller $\Delta y$ the top decay makes it difficult to resolve the initial direction of the $t \bar{t}$ system. Since we would not have this difficulty with the $b \bar{b}$ system, we might be less conservative and only assume $|y|<1$, in which case the cross section times acceptance rises to $1.0 \mathrm{pb}$, giving about $10^{4} b \bar{b}$ events.

Now we face more difficult questions - how accurately can we tag and measure the sign of one or both $b$ quarks, and what is the optimal procedure? A dedicated analysis will be necessary to answer these questions, so here we will limit ourselves to pointing out some of the issues and making some very preliminary estimates.

In an ideal world, it would be sufficient to simply find and measure the sign of a lepton inside of $b$-jets in dijet events. In the real world, this is complicated by the probability of semi-leptonic $b$ decays, the efficiency and mis-tagging rate of $b$-tagging, $B^{0}-\bar{B}^{0}$ mixing, and by the possibility of leptons from charm decays inside of $b$-jets (resulting in mismeasurements of the sign of the $b$ quark). These confounding factors might or might not make it worthwhile to require a $b$-tag for both jets, or perhaps to attempt to measure the sign of both jets in order to veto like-sign events. Let us consider these effects in turn. The probability of a semi-leptonic (electron or muon) $b$-decay is about 0.2 , and presumably this will be correlated with the $b$-tagging efficiency. The $b$-tagging efficiency at CDF peaks at $p_{T} \approx 60 \mathrm{GeV}$ and then slowly drops off at high $p_{T}$, falling to about 0.3 at $200 \mathrm{GeV}$ [60]. A

\footnotetext{
${ }^{3}$ The $b$-tagging efficiency at D0 gradually decreases for $|y|>1$ as can be seen from figure 28 of ref. [59]. Therefore, D0 may have a better sensitivity for the $b$-quark forward backward asymmetry.
} 
given $b$-quark will hadronize into a $B_{d}$ meson about $40 \%$ of the time, and into a $B_{s}$ meson about $11 \%$ of the time; other hadronization products do not mix with their anti-particles. So in total, a given high-energy $b$-quark will have a probability of about 0.13 to mix into a $\bar{B}$ meson before it decays, and this effect will dilute our sample. Many analyses address the issue of separating the decays of $D$ and $K$ mesons from $B$ decays for the purpose of studying these mesons. It should be possible to separate the leptons from $B$ decays from the leptons from charm decays for the purpose $b$-quark signing at high energies [4] using a cut on the lepton's momentum transverse to the jet (see figure 22 of ref. [59] for the muon $p_{T}$ distributions from $b$-quark and $c$-quark). Several studies, such as [61, 62], address the separation of charm and bottom mesons.

In the most optimistic scenario, it would be feasible to require only one $b$-tag. Then one would look for a lepton in either jet and tag its sign; the most optimistic probability for finding a lepton would be $\sim 30 \%$. The presence of this lepton should help to further reject non- $b$ dijet events. If we make the conservative assumption that $b$-tagging is independent of lepton-finding, then the overall acceptance of the tagging procedure will be about $10 \%$. With the requirement that $M_{b \bar{b}}>450 \mathrm{GeV},|y|<1$ for both jets, and $\Delta y>1$ we would be working with about 300 events. If these events have the same parton-level asymmetry as was observed for top quarks, namely $A_{F B}^{t \bar{t}} \approx 0.45$, then after convolving with the inevitable $B^{0}-\bar{B}^{0}$ mixing, we could expect about 200 events with a positive asymmetry and 100 events with a negative asymmetry. Relaxing the $\Delta y$ requirement would increase the total number of events by a factor of 3 , but it would dilute the asymmetry by an unknown amount. The largest and perhaps the most challenging unknown is the probability of fake wrongsign leptons from charm decays inside of $b$-jets. Even if a study with $M_{b \bar{b}}>450 \mathrm{GeV}$ proves very challenging, it is natural to hope that a study of lower energy $b \bar{b}$ events will be feasible, because the total cross section would be far larger and the $b$-tagging efficiency somewhat greater.

Requiring two $b$-tags would increase the purity of the sample, but it might be even more useful to require two leptons. At best this procedure accepts only $4 \%$ of $b \bar{b}$ events, but it makes it possible to measure the sign of both $b$-jets and reject like-sign events, reducing the impurities from leptons from charm decays and from $B^{0}-\bar{B}^{0}$ mixing. In the $t \bar{t}$ study it was possible to consider events with two $b$ tags as a cross check, so it is not unreasonable to think that a similar study will be possible in $b \bar{b}$.

A detailed experimental analysis will be necessary to settle these issues, but we are optimistic that CDF and D0 could measure the forward-backward asymmetry in $b \bar{b}$. Such a study would give us valuable insight into the nature of the $t \bar{t}$ asymmetry, and a positive result would greatly bolster the case for new physics.

\section{Discussion and conclusions}

In this paper we have performed a general study of color octet vector boson exchange as a possible source of the large top quark forward-backward asymmetry, $A_{F B}^{t \bar{t}}$, observed by $\mathrm{CDF}$ at high $t \bar{t}$ invariant masses. To set the tone for our subsequent discussions we first considered an effective field theory with only one new field, $G^{\prime}$, and examined the couplings 
that such a field would need in order to explain $A_{F B}^{t \bar{t}}$ while avoiding other experimental constraints arising from $(i)$ the $t \bar{t}$ total cross section as well as $t \bar{t}$ resonance searches at large $M_{t \bar{t}}$ at the Tevatron and, correspondingly, (ii) dijet resonance and contact interaction searches at the LHC. Given the large parameter freedom, we found that for a suitable choice of $G^{\prime}$ couplings all of these requirements can be simultaneously satisfied and would most likely lead to a rather wide state $(\Gamma / M>0.2)$ with a mass in excess of $\simeq 1.5 \mathrm{TeV}$. A state with such properties would very likely be found by various LHC searches performed during the coming year.

Problems arise, however, when we try to construct a detailed model. An obvious first choice would be a two-site coset model based on $\mathrm{SU}(3)_{1} \times \mathrm{SU}(3)_{2} / \mathrm{SU}(3)_{c}$. This scenario has only two free parameters, and while it was found to produce a large value for $A_{F B}^{t \bar{t}}$ with the correct sign, the values required for the light quark couplings were necessarily large enough to produce a significant signal for dijet contact interactions at the LHC, beyond that allowed by current CMS constraints. This model is, then, highly disfavored. We considered three-site generalizations, but they did not fare much better.

Both the two and three site models necessarily coupled the axigluon(s) with equal strength to the top and light quarks. Since we require a large coupling to the top quark to produce a large $A_{F B}^{t \bar{t}}$, these models necessarily led to large dijet contact interactions. Clearly, one needs to construct a model where the axigluon couplings to the top-quark can be large while maintaining small couplings to the light quarks; one way to do this was to return to the original two-site model and introduce a new $Q=2 / 3$ vector-like fermion which mixes with the $\mathrm{RH} u$-quark, introducing an additional free parameter. In this case we found that there were significant regions of parameter space that satisfied all of the experimental requirements with a rather light $G^{\prime}$ mass $\sim 1 \mathrm{TeV}$. The LHC is capable of probing all of this parameter space during the coming year. Furthermore, if the new vector-like fermion has a mass of $1-2 \mathrm{TeV}$, its pair-production and subsequent decay to $u+G^{\prime(*)} \rightarrow u+t \bar{t}, u+u \bar{u}$, with the $G^{\prime}$ being either on- or off-shell, could be visible at the LHC.

Since in all of these scenarios the third generation fermion couplings to the new color octet vector states differed from those of the first two, FCNCs could become problematic when one rotates to the mass eigenstate basis. We derived the general form for the constraints that arise from mixing in the Kaon, $D$ and $B_{q}$ meson sectors. These are made somewhat more complicated by the generic existence of both left- and right-handed FC interactions, necessitating a full operator analysis. However, it is impossible to numerically evaluate these FCNC contributions in a completely model-independent fashion since there are, in general, four distinct unitary matrices which are necessary to rotate the $Q=2 / 3$ and $-1 / 3$ fermions from the weak to the mass eigenstate basis and only the experimental values of the CKM matrix (for LH charged currents) act as direct constraints. To perform this evaluation in a unique way requires that any model under consideration also provides a theory of flavor (which ours do not). If we assume that the left-handed and right-handed mixing matrices are identical, we found that all the $A_{F B}^{t \bar{t}}$ preferred regions for the models considered here are excluded. However, if the two right-handed mixing matrices are trivial, many axigluon models survive the flavor constraints. 
Since $t_{L}$ and $b_{L}$ lie in a single doublet and any model with an $\mathrm{SU}(2)_{R}$ custodial symmetry relates $t_{R}$ and $b_{R}$ couplings, it is natural to ask if the asymmetry observed in the $t \bar{t}$ system might also manifest itself in the $b \bar{b}$ final state at large invariant masses at the Tevatron or the LHC. Given the level of statistics and the issues associated with $b$-tagging and signing plus possible dilutions arising from mixing and charm contamination it is not possible for us to easily evaluate how feasible such measurements might be. We encourage the collider collaborations to consider this possibility, especially if the large values of $A_{F B}^{t \bar{t}}$ are confirmed by future measurements. Clearly this would provide us with valuable information on the physics behind this asymmetry.

We have constructed models of color octet vector bosons that explain the large value of $A_{F B}^{t \bar{t}}$ while remaining consistent with collider and flavor constraints, but it should be clear from the discussion above that they are not particularly elegant. All of these models necessarily entail dijet or $t \bar{t}$ signatures that will be testable in the coming year at the LHC. An immediate experimental prospect is worth a thousand models.

\section{Acknowledgments}

We would like to thank Dan Amidei, Brendan Casey, Tim Tait, and Jay Wacker for useful discussions and comments. We also want to thank Tom LeCompte and Henri Bachacou for discussing the ATLAS dijet contact interaction limit. SLAC is operated by Stanford University for the US Department of Energy under contract DE-AC02-76SF00515.

Open Access. This article is distributed under the terms of the Creative Commons Attribution Noncommercial License which permits any noncommercial use, distribution, and reproduction in any medium, provided the original author(s) and source are credited.

\section{References}

[1] CDF collaboration, T. Aaltonen et al., Evidence for a mass dependent forward-backward asymmetry in top quark pair production, arXiv:1101.0034 [SPIRES].

[2] CDF collaboration, T. Aaltonen et al., Forward-backward asymmetry in top quark production in p p collisions at $\sqrt{s}=1.96$ TeV, Phys. Rev. Lett. 101 (2008) 202001 [arXiv: 0806.2472] [SPIRES].

[3] D0 collaboration, V.M. Abazov et al., First measurement of the forward-backward charge asymmetry in top quark pair production, Phys. Rev. Lett. 100 (2008) 142002 [arXiv: 0712.0851] [SPIRES].

[4] L.M. Sehgal and M. Wanninger, Forward-backward asymmetry in two jet events: signature of axigluons in proton-anti-proton collisions, Phys. Lett. B 200 (1988) 211 [SPIRES].

[5] J. Bagger, C. Schmidt and S. King, Axigluon production in hadronic collisions, Phys. Rev. D 37 (1988) 1188 [SPIRES].

[6] M.V. Martynov and A.D. Smirnov, Chiral color symmetry and possible $G^{\prime}$-boson effects at the Tevatron and LHC, Mod. Phys. Lett. A 24 (2009) 1897 [arXiv:0906.4525] [SPIRES].

[7] S. Jung, H. Murayama, A. Pierce and J.D. Wells, Top quark forward-backward asymmetry from new t-channel physics, Phys. Rev. D 81 (2010) 015004 [arXiv:0907.4112] [SPIRES]. 
[8] P. Ferrario and G. Rodrigo, Constraining heavy colored resonances from top-antitop quark events, Phys. Rev. D 80 (2009) 051701 [arXiv:0906.5541] [SPIRES].

[9] K. Cheung, W.-Y. Keung and T.-C. Yuan, Top quark forward-backward asymmetry, Phys. Lett. B 682 (2009) 287 [arXiv:0908.2589] [SPIRES].

[10] D.-W. Jung, P. Ko, J.S. Lee and S.-H. Nam, Model independent analysis of the forward-backward asymmetry of top quark production at the Tevatron, Phys. Lett. B 691 (2010) 238 [arXiv:0912.1105] [SPIRES].

[11] R.S. Chivukula, E.H. Simmons and C.P. Yuan, Axigluons cannot explain the observed top quark forward-backward asymmetry, Phys. Rev. D 82 (2010) 094009 [arXiv:1007.0260] [SPIRES].

[12] Q.-H. Cao, D. McKeen, J.L. Rosner, G. Shaughnessy and C.E.M. Wagner, Forward-backward asymmetry of top quark pair production, Phys. Rev. D 81 (2010) 114004 [arXiv:1003.3461] [SPIRES].

[13] M.V. Martynov and A.D. Smirnov, On mass limit for chiral color symmetry $G^{\prime}$-boson from Tevatron data on $t \bar{t}$ production, Mod. Phys. Lett. A 25 (2010) 2637 [arXiv:1006.4246] [SPIRES].

[14] D. Choudhury, R.M. Godbole, S.D. Rindani and P. Saha, Top polarization, forward-backward asymmetry and new physics, arXiv:1012.4750 [SPIRES].

[15] K. Cheung and T.-C. Yuan, Top quark forward-backward asymmetry in the large invariant mass region, arXiv: 1101.1445 [SPIRES].

[16] D.E. Morrissey, T. Plehn and T.M.P. Tait, Physics searches at the LHC, arXiv:0912.3259 [SPIRES].

[17] C.T. Hill, Topcolor: Top quark condensation in a gauge extension of the standard model, Phys. Lett. B 266 (1991) 419 [SPIRES].

[18] C.T. Hill and S.J. Parke, Top production: sensitivity to new physics, Phys. Rev. D 49 (1994) 4454 [hep-ph/9312324] [SPIRES].

[19] K.D. Lane and M.V. Ramana, Walking technicolor signatures at hadron colliders, Phys. Rev. D 44 (1991) 2678 [SPIRES].

[20] L. Randall and R. Sundrum, A large mass hierarchy from a small extra dimension, Phys. Rev. Lett. 83 (1999) 3370 [hep-ph/9905221] [SPIRES].

[21] H. Davoudiasl, J.L. Hewett and T.G. Rizzo, Bulk gauge fields in the Randall-Sundrum model, Phys. Lett. B 473 (2000) 43 [hep-ph/9911262] [SPIRES].

[22] T. Appelquist, H.-C. Cheng and B.A. Dobrescu, Bounds on universal extra dimensions, Phys. Rev. D 64 (2001) 035002 [hep-ph/0012100] [SPIRES].

[23] J.C. Pati and A. Salam, Are the new particles color gluons?, Phys. Rev. Lett. 34 (1975) 613 [SPIRES].

[24] L.J. Hall and A.E. Nelson, Heavy gluons and monojets, Phys. Lett. B 153 (1985) 430 [SPIRES].

[25] P.H. Frampton and S.L. Glashow, Chiral color: an alternative to the standard model, Phys. Lett. B 190 (1987) 157 [SPIRES].

[26] J.L. Hewett and T.G. Rizzo, Low-energy phenomenology of superstring inspired $E_{6}$ models, Phys. Rept. 183 (1989) 193 [SPIRES]. 
[27] M. Cacciari, S. Frixione, M.L. Mangano, P. Nason and G. Ridolfi, Updated predictions for the total production cross sections of top and of heavier quark pairs at the Tevatron and at the LHC, JHEP 09 (2008) 127 [arXiv:0804.2800] [SPIRES].

[28] CDF collaboration, Combination of CDF top quark pair production cross-section measurements with up to $4.6 \mathrm{fb}^{-1}$, CDF public note 9913 (2009).

[29] D0 collaboration, V.M. Abazov et al., Search for $t \bar{t}$ resonances in the lepton plus jets final state in $p \bar{p}$ collisions at $\sqrt{s}=1.96 \mathrm{TeV}$, Phys. Lett. B 668 (2008) 98 [arXiv:0804.3664] [SPIRES].

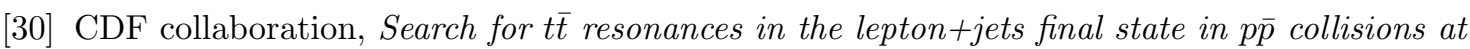
$\sqrt{s}=1.96 \mathrm{TeV}$, D conference note $5882(2009)$.

[31] CDF collaboration, Search for resonant $t \bar{t}$ production in p p $\bar{p}$ collisions at $\sqrt{s}=1.96 \mathrm{TeV}, \mathrm{CDF}$ public note 9844 (2009).

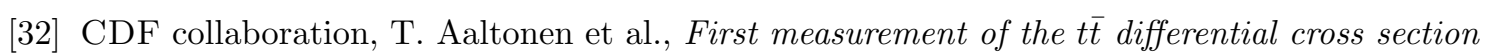
$d \sigma / d M_{t \bar{t}}$ in p p collisions at $\sqrt{s}=1.96 \mathrm{TeV}$, Phys. Rev. Lett. 102 (2009) 222003 [arXiv:0903.2850] [SPIRES].

[33] CDF collaboration, T. Aaltonen et al., Search for new color-octet vector particle decaying to $t \bar{t}$ in p p collisions at $\sqrt{s}=1.96$ TeV, Phys. Lett. B 691 (2010) 183 [arXiv:0911.3112] [SPIRES].

[34] CMS collaboration, V. Khachatryan et al., Search for dijet resonances in $7 \mathrm{TeV} \mathrm{pp}$ Collisions at CMS, Phys. Rev. Lett. 105 (2010) 211801 [arXiv:1010.0203] [SPIRES].

[35] T. Han, I. Lewis and Z. Liu, Colored resonant signals at the LHC: largest rate and simplest topology, JHEP 12 (2010) 085 [arXiv: 1010.4309] [SPIRES].

[36] ATLAS collaboration, G. Aad et al., Search for quark contact interactions in dijet angular distributions in pp collisions at $\sqrt{s}=7 \mathrm{TeV}$ measured with the ATLAS detector, Phys. Lett. B 694 (2011) 327 [arXiv:1009.5069] [SPIRES].

[37] J. Gao, C.S. Li, J. Wang, H.X. Zhu and C.P. Yuan, Next-to-leading QCD effect to the quark compositeness search at the LHC, arXiv:1101.4611 [SPIRES].

[38] P.H. Frampton, J. Shu and K. Wang, Axigluon as possible explanation for $p \bar{p} \rightarrow t \bar{t}$ forward-backward asymmetry, Phys. Lett. B 683 (2010) 294 [arXiv:0911.2955] [SPIRES].

[39] Y. Bai and B.A. Dobrescu, Heavy octets and Tevatron signals with three or four $b$ jets, arXiv: 1012.5814 [SPIRES].

[40] UTFIT collaboration, M. Bona et al., Model-independent constraints on $\Delta F=2$ operators and the scale of new physics, JHEP 03 (2008) 049 [arXiv:0707.0636] [SPIRES].

[41] M. Blanke, A.J. Buras, B. Duling, S. Gori and A. Weiler, $\Delta F=2$ observables and fine-tuning in a warped extra dimension with custodial protection, JHEP 03 (2009) 001 [arXiv:0809.1073] [SPIRES].

[42] E. Golowich, J. Hewett, S. Pakvasa and A.A. Petrov, Implications of $D^{0}-\bar{D}^{0}$ mixing for new physics, Phys. Rev. D 76 (2007) 095009 [arXiv:0705.3650] [SPIRES].

[43] Particle Data Group collaboration, K. Nakamura et al., Review of particle physics, J. Phys. G 37 (2010) 075021 [SPIRES].

[44] CMS collaboration, V. Khachatryan et al., First measurement of the cross section for top-quark pair production in proton-proton collisions at $\sqrt{s}=7$ TeV, Phys. Lett. B 695 (2011) 424 [arXiv: 1010.5994] [SPIRES]. 
[45] The ATLAS collaboration, G. Aad et al., Expected performance of the ATLAS experiment - Detector, trigger and physics, arXiv:0901.0512 [SPIRES].

[46] E. Cogneras and D. Pallin, Generic t $\bar{t}$ resonance search with the ATLAS detector, ATLPHYS-PUB-2006-033 (2006).

[47] V. Barger, T. Han and D.G.E. Walker, Top quark pairs at high invariant mass: a model-independent discriminator of new physics at the LHC, Phys. Rev. Lett. 100 (2008) 031801 [hep-ph/0612016] [SPIRES].

[48] D. Choudhury, R.M. Godbole, R.K. Singh and K. Wagh, Top production at the Tevatron/LHC and nonstandard, strongly interacting spin one particles, Phys. Lett. B 657 (2007) 69 [arXiv: 0705.1499] [SPIRES].

[49] U. Baur and L.H. Orr, Searching for $t \bar{t}$ resonances at the Large Hadron Collider, Phys. Rev. D 77 (2008) 114001 [arXiv:0803.1160] [SPIRES].

[50] Y. Bai and Z. Han, Top-antitop and top-top resonances in the dilepton channel at the CERN LHC, JHEP 04 (2009) 056 [arXiv:0809.4487] [SPIRES].

[51] D.E. Kaplan, K. Rehermann, M.D. Schwartz and B. Tweedie, Top tagging: a method for identifying boosted hadronically decaying top quarks, Phys. Rev. Lett. 101 (2008) 142001 [arXiv:0806.0848] [SPIRES].

[52] T. Plehn, M. Spannowsky, M. Takeuchi and D. Zerwas, Stop reconstruction with tagged tops, JHEP 10 (2010) 078 [arXiv: 1006.2833] [SPIRES].

[53] K. Rehermann and B. Tweedie, Efficient identification of boosted semileptonic top quarks at the LHC, arXiv:1007.2221 [SPIRES].

[54] A. Abdesselam et al., Boosted objects: a probe of beyond the standard model physics, arXiv:1012.5412 [SPIRES].

[55] R. Frederix and F. Maltoni, Top pair invariant mass distribution: a window on new physics, JHEP 01 (2009) 047 [arXiv: 0712.2355] [SPIRES].

[56] S. Moch and P. Uwer, Theoretical status and prospects for top-quark pair production at hadron colliders, Phys. Rev. D 78 (2008) 034003 [arXiv:0804.1476] [SPIRES].

[57] A.D. Martin, W.J. Stirling, R.S. Thorne and G. Watt, Parton distributions for the LHC, Eur. Phys. J. C 63 (2009) 189 [arXiv:0901.0002] [SPIRES].

[58] CDF collaboration, D.E. Acosta et al., Measurement of the $t \bar{t}$ production cross section in $p \bar{p}$ collisions at $\sqrt{s}=1.96 \mathrm{TeV}$ using lepton plus jets events with semileptonic B decays to muons, Phys. Rev. D 72 (2005) 032002 [hep-ex/0506001] [SPIRES].

[59] The D0 collaboration, V.M. Abazov et al., b-Jet Identification in the D0 experiment, Nucl. Instrum. Meth. A 620 (2010) 490 [arXiv:1002.4224] [SPIRES].

[60] CDF collaboration, C. Neu, CDF b-tagging: measuring efficiency and false positive rate, FERMILAB-CONF-06-162-E [SPIRES].

[61] D0 collaboration, V.M. Abazov et al., Measurement of $B_{d}$ mixing using opposite-side flavor tagging, Phys. Rev. D 74 (2006) 112002 [hep-ex/0609034] [SPIRES].

[62] CDF collaboration, T. Aaltonen et al., Measurements of branching fraction ratios and CP asymmetries in $B^{ \pm} \rightarrow D_{C P} K^{ \pm}$decays in hadron collisions, Phys. Rev. D 81 (2010) 031105 [arXiv:0911.0425] [SPIRES]. 